\title{
Synergistic effects of liposomes encapsulating atorvastatin calcium and curcumin and targeting dysfunctional endothelial cells in reducing atherosclerosis
}

This article was published in the following Dove Medical Press journal:

International Journal of Nanomedicine

\begin{abstract}
Xiaoxia Li, ',* Hong Xiao, ',* Chaowen Lin, 2,3,* Weitong Sun,' Teng Wu,' Jin Wang, ${ }^{4}$ Bin Chen, ${ }^{3}$ Xia Chen, ${ }^{2}$ Du Cheng'

'PCFM Lab of Ministry of Education, School of Materials Science and Engineering, Sun Yat-sen University, Guangzhou 5I0275, People's Republic of China; ${ }^{2}$ Department of Cardiovascular Medicine, The Third Affiliated Hospital, Southern Medical University, Guangzhou 5 I0630, People's Republic of China; ${ }^{3}$ Department of Orthopedics and Traumatology, Nanfang Hospital, Southern Medical University, Guangzhou 5105I5, People's Republic of China; ${ }^{4}$ Department of Radiology, The Third Affiliated Hospital, Sun Yat-sen University, Guangzhou 510630, People's Republic of China

*These authors contributed equally to this work
\end{abstract}

Correspondence: Du Cheng PCFM Lab of Ministry of Education, School of Materials Science and Engineering, Sun Yat-sen University, Guangzhou 510275 , People's Republic of China

Tel $+862084 I I 2172$

Fax +86 20 84II 2245

Email chengdu@mail.sysu.edu.cn

Xia Chen

Department of Cardiovascular Medicine, The Third Affiliated Hospital, Southern Medical University, Guangzhou 510630 , People's Republic of China

Tel +86 2062784710

$\mathrm{Fax}+862062784710$

Email amber23@smu.edu.cn
Background: Atherosclerosis is a major cardiovascular disease that causes ischemia of the heart, brain, or extremities, and can lead to infarction. The hypolipidemic agent atorvastatin calcium (Ato) alleviates atherosclerosis by reducing plasma lipid and inflammatory factors. However, the low bioavailability of Ato limits its widespread use and clinical effectiveness. Curcumin (Cur), a natural polyphenol with antioxidation and anti-inflammation bioactivities, has potential anti-atherosclerosis activity and may reduce Ato-induced cytotoxicity.

Materials and methods: Liposomes modified using a targeting ligand (E-selectin-binding peptide) were prepared to co-deliver Ato and Cur to dysfunctional endothelial cells (ECs) overexpressing E-selectin. Molecules involved in the inhibition of adhesion (E-selectin and intercellular cell adhesion molecule-1 [ICAM-1]) and inflammation (IL-6 and monocyte chemotactic protein 1 [MCP-1]) in human aortic endothelial cells were evaluated using real-time quantitative PCR, flow cytometry, and immunofluorescence staining. The antiatherosclerosis effects of liposomes co-loaded with Ato and Cur in vivo were evaluated using ApoE knockout $\left(\mathrm{ApoE}^{--}\right)$mice.

Results: Targeted liposomes delivered Ato and Cur to dysfunctional ECs, resulting in synergistic suppression of adhesion molecules (E-selectin and ICAM-1) and plasma lipid levels. Moreover, this treatment reduced foam cell formation and the secretion of inflammatory factors (IL-6 and MCP-1) by blocking monocyte migration into the intima. In addition, Cur successfully reduced Ato-inducible cytotoxicity.

Conclusion: Both in vitro and in vivo experiments demonstrated that cell-targeted co-delivery of Ato and Cur to dysfunctional ECs drastically reduces atherosclerotic lesions with fewer side effects than either Ato or Cur alone.

Keywords: combined therapy, atorvastatin calcium, curcumin, antiatherosclerosis, targeted codelivery

\section{Introduction}

Atherosclerosis, a major cardiovascular disease, causes ischemia of the heart, brain, or extremities, and even leads to infarction. ${ }^{1}$ During atherosclerosis progression, the damaged endothelium secretes adhesion molecules (eg, intercellular cell adhesion molecule-1 [ICAM-1], E-selectin, and P-selectin) to capture monocytes, resulting in the formation of foam cells, which recruit more monocytes via secretion of IL-6 to stimulate endothelial cells (ECs) production of chemokines (eg, monocyte chemotactic protein 1 [MCP-1] and CXC chemokine receptor 2). ${ }^{2,3}$ Thus, the pathology of atherosclerosis can be ascribed to inflammatory processes. ${ }^{4,5}$ 
Recently, the inflamed endothelium has become a focus for the development of anti-atherosclerosis therapies and several drugs have been developed to treat hyperlipidemia and/or inflamed ECs. For example, statin-like drugs, such as atorvastatin, simvastatin, and lovastatin, have been used to reduce the plasma lipids by inhibiting 3-hydroxy-3-methyl-glutaryl CoA (HMG-CoA) reductase. ${ }^{6}$ Atorvastatin calcium (Ato) led to superior anti-atherosclerosis outcome in a randomized clinical trial by reducing plasma lipids and inhibiting intimal smooth muscle cell accumulation, as well as reducing intimal hyperplasia and promoting intimal regeneration. ${ }^{7,8}$ Nevertheless, Ato-inducible side effects (eg, CoQ10-lowering effect, diabetes mellitus, and central nervous system complaints) limit the clinical application of this treatment,, 10 and many methods of reducing the side effects of statins by combining them with curcumin (Cur), coenzyme Q10, vitamin D, and artichoke have been investigated. ${ }^{11-13}$ Among these approaches, Cur, a polyphenol derived from the rhizome of the plant Curcuma longa, has multiple bioactivities, including antioxidation, anti-inflammatory, anticancer, and thrombosis suppression effects, ${ }^{14-16}$ and functions by reducing plasma triglycerides (TGs) and increasing high-density lipoprotein cholesterol levels. ${ }^{17,18}$ Cur effectively inhibited the development of atherosclerosis in ApoE/LDLR double knockout mice via its anti-inflammatory and antioxidant activities. ${ }^{19}$ Notably, a recent study reported that Cur could substantially relieve the statin-associated symptom (SAS). ${ }^{10}$ Therefore, we hypothesized that combination therapy with Ato and Cur could synergistically reduce atherosclerosis and alleviate side effects of Ato.

Both hydrophilic statins (eg, rosuvastatin) and lipophilic statins (eg, atorvastatin) can prevent cardiovascular disease by reducing plasma endothelin- $1^{20}$ and asymmetric dimethylarginine ${ }^{21}$ levels, respectively, resulting in similar therapeutic outcomes; however, these treatments have some limitations, such as low bioavailability, adverse effects, and nonspecific delivery. Therefore, several strategies have been developed to further improve the anti-atherosclerosis effects of statins. First, nanomedicine-based strategy provides a powerful platform to enhance bioavailability of hydrophobic drugs. ${ }^{22}$ For example, Cur, ${ }^{23}$ Ato, ${ }^{24}$ and simvastatin ${ }^{25}$ exhibited higher anticancer and osteogenesis effects when encapsulated into dextran-nanoceria, polymeric micelles, or liposomes, respectively, rather than applied in their free forms. Liposomes, assembled from lipid bilayers, have been extensively applied for drug delivery because they are biocompatible, biodegradable, feasible, and exhibit high transfection efficiencies. ${ }^{25-27}$ Second, co-delivery strategies, using multifunctional nanocarriers, have been used to induce synergistic therapeutic effects and alleviate side effects. ${ }^{28}$ Third, targeting ligands have been used to enable cell-specific delivery of drugs. ${ }^{29,30}$ For example, liposomes decorated with targeting ligand (eg, folate or cyclic Arg-GlyAsp [RGD] peptide) can effectively deliver drug into the nidus position using an active targeting delivery mechanism, resulting in the desired therapeutic effects and minimal side effects. ${ }^{31-34}$ Notably, integration of co-delivery and targeted delivery in one nanosystem could significantly strengthen anti-atherosclerosis effects and reduce adverse effects. To achieve targeted delivery of Ato and Cur to inflamed/ dysfunctional ECs, target epitopes, specifically upregulated on the surface of diseased ECs, can be targeted by specific antibody-conjugated nanomedicines, effectively shuttling cargos into inflamed ECs. ${ }^{35}$ Cell adhesion molecules, such as the E-selectin protein, are ideal target epitopes, because of their restricted overexpression on inflamed ECs and effective internalization following ligand binding. ${ }^{36}$ For example, an E-selectin-modified liposome was used to effectively deliver siRNA to inflamed ECs to inhibit vascular endothelialcadherin gene expression. ${ }^{37-39}$

Although the liposomes and polymeric nanoparticles have been employed to deliver Ato or Cur alone, ${ }^{40,41}$ the codelivery of Ato and Cur has rarely been applied as an antiatherosclerosis treatment. Specifically, our delivery strategy of targeting inflamed ECs, rather than macrophages, may provide an alternative approach, acting at an early stage of atherosclerosis by blocking the adhesion of monocytes and the transition of macrophages into foam cells (Figure 1). Herein, we conducted both in vitro and in vivo experiments to investigate the anti-atherosclerotic effects of the combination therapy of Ato and Cur.

\section{Experimental section Materials}

Cholesterol (Chol, Mw: 386.65) in powder form, 1,2-dipalmitoyl-sn-glycero-3-phosphocholine (DPPC, Mw: 734.05), and 1,2-distearoyl-sn-glycero-3-phosphoethanolamine-N(maleimide(polyethylene glycol) ${ }_{2000}$ ) (Mal- $\mathrm{PEG}_{2000}$-DSPE, Mw: 2893) were purchased from Avanti Polar Lipids Inc. (Alabaster, AL, USA). Ato and Cur were purchased from Sigma-Aldrich (St Louis, MO, USA). E-selectin-binding (Esb) peptide (sequence: $\mathrm{H}_{2} \mathrm{~N}$-DITWDQLWDLMK$\mathrm{COOH}$ ) was synthesized by Minghao Biotech Inc. Co. (Wuhan, China). Flow cytometer and real-time mRNA quantification kits were purchased from KeyGen Biotech In. Co. (Nanjing, China) and Roche Life Science (Basel, 


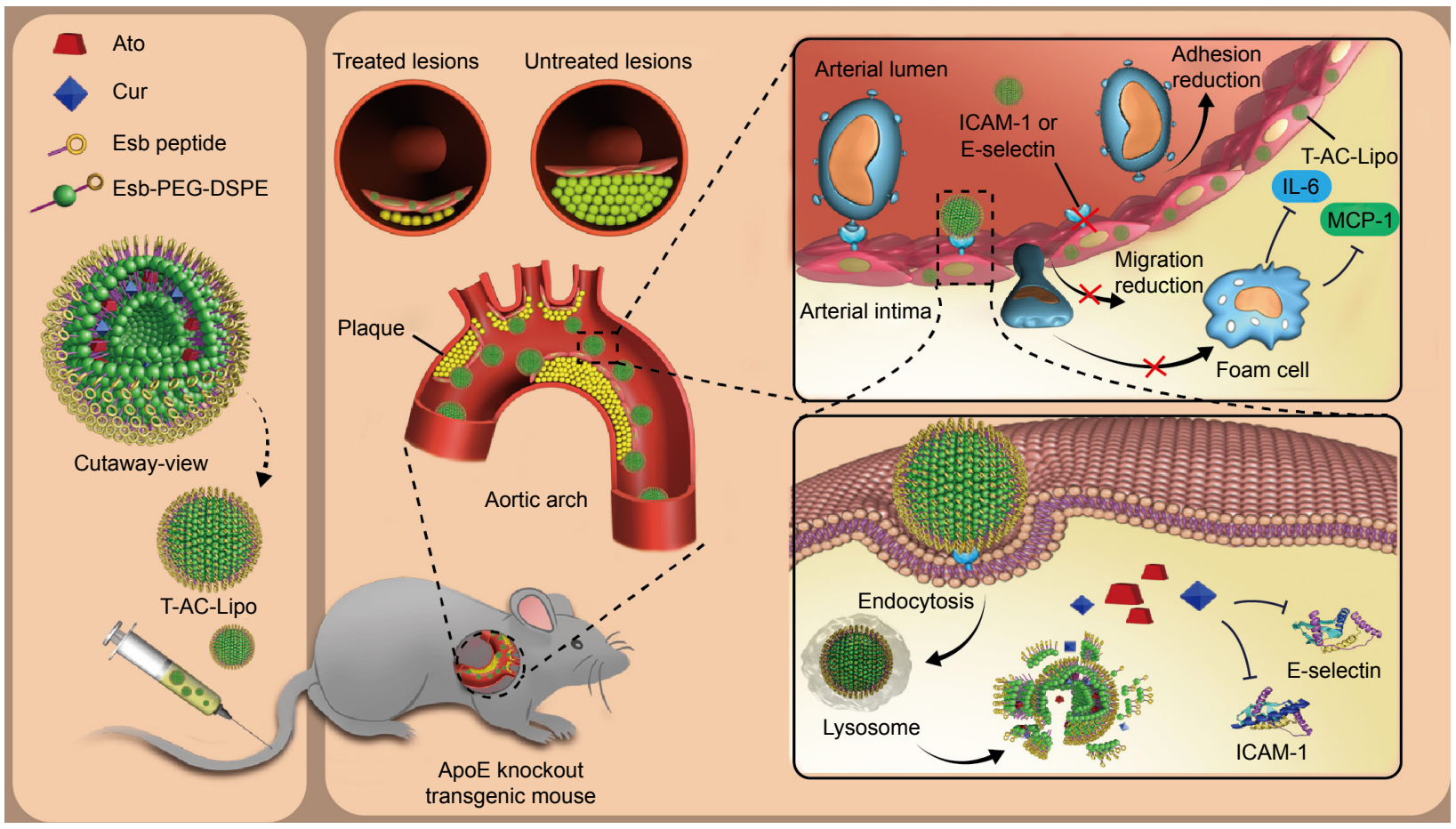

Figure I Illustration of E-selectin-targeting liposomes (T-AC-Lipo) simultaneously encapsulating Ato and Cur to treat atherosclerotic ApoE knockout mice. Abbreviations: Ato, atorvastatin calcium; Cur, curcumin; Esb peptide, E-selectin-binding peptide; ICAM-I, intercellular cell adhesion molecule-I; IL-6, interleukin 6; MCP-I, monocyte chemotactic protein I; T-AC-Lipo, targeting-ligand-modified atorvastatin calcium- and curcumin-loaded liposome.

Switzerland), respectively. The fluorescent probe, DAPI, was from Beyotime (Shanghai, China).

\section{Liposome preparation}

Liposomes loaded with Ato and Cur (AC-Lipo) were prepared using the thin-film hydration-sonication method. Briefly, $5 \mathrm{~mL}$ of phospholipids (20 mg DPPC, $4 \mathrm{mg}$ Chol, and $4 \mathrm{mg}$ DSPE-PEG), Ato (1 mg), and Cur (1 mg) dissolved in chloroform were transferred into a $100 \mathrm{~mL}$ pear-shape flask with a $2.4 \mathrm{~cm}$ bottle mouth to prepare a thin film under rotating evaporation. To prepare AC-Lipo, film hydrated with $10 \mathrm{~mL}$ of $10 \mathrm{mM}$ PBS ( $\mathrm{pH} \mathrm{7.4)} \mathrm{was} \mathrm{maintained} \mathrm{at} 37^{\circ} \mathrm{C}$ in a shaking incubator for 1 hour. The liposome suspension was sonicated using an ultrasonic processor (VCS $130 \mathrm{~PB}$, Sonics, USA; $130 \mathrm{~W}, 5$ minutes), followed by filtration through a $450 \mathrm{~nm}$ pore-size filter. The resultant liposomes were maintained at $4{ }^{\circ} \mathrm{C}$ for future use. Blank liposomes (B-Lipo), without Ato and Cur, were prepared in the same way. Esb peptide-PEG-DSPE was substituted for PEG-DSPE during preparation of EC-targeted liposomes. The Esb peptide was conjugated with Mal-decorated PEG-DSPE ( $0.25 \mathrm{~g}$, $0.1 \mathrm{mmol}$ ) using a click reaction between sulfhydryl and maleimide. In brief, a solution of Mal-PEG-DSPE vesicles in $10 \mathrm{mM}$ PBS (pH 7.4) and Esb peptide (0.2 mmol, 2 molar equivalents of Mal) in $2 \mathrm{~mL} \mathrm{PBS}$ ( $\mathrm{pH} 7.4$ ) were mixed by continuous stirring at room temperature for 10 hours. Excess Esb peptide (molecular weight cutoff: $14 \mathrm{kDa}$ ) was removed by dialyzing against PBS ( $\mathrm{pH} 7.4$ ) for 2 days.

Ato and Cur drug-loading content (DLC), defined as the percentage weight of Ato and Cur in the liposomes, was quantified using a UV-Vis Spectrophotometer (UV-3150, Shimadzu, Japan). After lyophilization, the weight of the liposomes was determined. To generate calibration curves (absorbance against concentration), the absorbance of Cur $(425 \mathrm{~nm})$ and Ato $(246 \mathrm{~nm})$ in $\mathrm{MeOH}$ was measured at various concentrations. Freeze-dried liposome samples (5 mg) were redissolved in $\mathrm{MeOH}(1.0 \mathrm{~mL})$ to measure the absorbance of Ato and Cur at 425 and $246 \mathrm{~nm}$, respectively. The concentrations of Ato and Cur in the liposomes were calculated using the previously constructed calibration curves. Furthermore, the absolute masses of the drugs in solution were calculated by multiplying the concentration by the solution volume. The DLC and drug-loading efficiency (DLE) values of liposomes were calculated using the following formulae:

DLC $=$ Absolute mass of drug in solution/absolute mass of liposome in solution $\times 100 \%$

DLE $=$ Absolute mass of drug in total liposome/absolute mass of drug in feed $\times 100 \%$. 


\section{Liposome characterization}

Transmission electron microscopy (TEM) imaging was performed using a Hitachi Model H 7650 (Hitachi, Co., Ltd., Tokyo, Japan); power, $80 \mathrm{kV}$. To prepare a sample for TEM, a $5 \mu \mathrm{L}(1 \mathrm{mg} / \mathrm{mL})$ aliquot was dried on a copper grid decorated with amorphous carbon and a small drop of uranyl acetate solution ( $2 \mathrm{wt} \%$ in water) was applied to stain the sample. After incubation for 60 seconds, the stain was blotted with a filter paper. Finally, grids were dried overnight in a desiccator, then subjected to TEM imaging. The zeta potential and hydrodynamic size of liposomes were measured by dynamic light scattering (DLS) at $25^{\circ} \mathrm{C}$ on Zetasizer Nano ZS equipment (Malvern, UK) with scattered light detection angles of $90^{\circ}$ and $15^{\circ}$, respectively.

\section{Cell viability}

Human aortic endothelial cells (HAECs) were purchased form BeNa Culture Collection Co. (Beijing, China) and routinely cultured in tissue culture flasks (Corning, CA, USA) at $37^{\circ} \mathrm{C}$ with $5 \% \mathrm{CO}_{2}$ in RPMI 1640 medium containing $10 \%$ FBS and 1\% antibiotics (Penicillin-Streptomycin). All cell culture reagents were sourced from Gibco (Life Technologies, Grand Island, NY, USA). When HAECs reached $80 \%$ confluence, they were detached from flasks with $0.25 \%$ trypsin, seeded into 96-well plates at a density of $5 \times 10^{3}$ cells per well, and cultured overnight with $5 \% \mathrm{CO}_{2}$ at $37^{\circ} \mathrm{C}$ under a humidified atmosphere. After incubation with curcuminloaded liposome (C-Lipo), atorvastatin calcium-loaded liposome (A-Lipo), AC-Lipo, and Esb peptide-modified ACLipo (T-AC-Lipo) at different concentrations for 48 hours, culture medium was replaced with $100 \mu \mathrm{L}$ fresh RPMI 1640 containing $10 \mu \mathrm{L}$ MTT ( $5 \mathrm{mg} / \mathrm{mL}$ MTT in PBS). Then, plates were incubated at $37^{\circ} \mathrm{C}$ for a further 4 hours. After discarding MTT-containing medium, dimethyl sulfoxide was added (100 $\mu \mathrm{L}$ per well) to dissolve the precipitate and absorbance at $570 \mathrm{~nm}$ measured using a Microplate Reader (Sunrise ${ }^{\mathrm{TM}}$, Tecan, Switzerland).

\section{Confocal laser scanning microscopy (CLSM)}

Cell uptake of targeting and nontargeting liposomes containing Cur was imaged using an LSM 710 microscope (Carl Zeiss Inc., Baden-Wurttemberg, Germany). HAEC cells $\left(1 \times 10^{3}\right)$ were seeded in confocal dishes with glass bases. After overnight incubation at $37^{\circ} \mathrm{C}$, cells were pretreated with $1 \mu \mathrm{g} / \mathrm{mL}$ lipopolysaccharide (LPS) for 4 hours and then incubated with AC-Lipo, with or without Esb peptide modification, at a Cur concentration of $7.2 \mu \mathrm{g} / \mathrm{mL}$. After washing twice with PBS, cells were fixed in $4 \%$ paraformaldehyde for 10 minutes. Nuclei were stained with DAPI $(1 \mu \mathrm{g} / \mathrm{mL}$ in PBS) for 15 minutes, then Cur and DAPI fluorescence emission data collected at 530 and $460 \mathrm{~nm}$, with laser excitation at 488 and $405 \mathrm{~nm}$, respectively.

For immunofluorescence assays of relative protein (ICAM-1 and E-selectin) expression, cells fixed in 4\% paraformaldehyde were sequentially incubated with ICAM-1 and E-selectin monoclonal antibodies (Abcam Co., Ltd., Cambridge, UK). After washing three times with PBS, cells were further incubated with fluorescent dye (AF555)labeled secondary antibody (Abcam Co., Ltd., Cambridge, UK). Nuclei were stained with DAPI. AF555 and AF647 fluorescence (excitation, 552 and $638 \mathrm{~nm}$; emission, 570 and $665 \mathrm{~nm}$, respectively) were recorded to evaluate E-selectin and ICAM-1 protein levels.

\section{Flow cytometry}

HAECs were plated in six-well plates $\left(3 \times 10^{5}\right.$ cells per well) and incubated with RPMI 1640 medium at $37^{\circ} \mathrm{C}$ under a humidified atmosphere containing $5 \% \mathrm{CO}_{2}$. Before transfection with various liposomes, cells were preincubated with $1 \mu \mathrm{g} / \mathrm{mL}$ LPS for 4 hours and then treated for 12 hours at $37^{\circ} \mathrm{C}$ with fresh medium containing AC-Lipo, T-AC-Lipo, or T-AC-Lipo plus Esb (preincubation with Esb before T-AC-Lipo treatment), at a Cur concentration of $7.2 \mu \mathrm{g} / \mathrm{mL}$. After washing three times with PBS, cells were harvested by trypsinization and centrifugation (1,000 rpm, 5 minutes). Finally, cells were resuspended in $500 \mu \mathrm{L}$ PBS for further analysis. Transfection efficiency was measured using a flow cytometer (Gallios, Beckman Coulter, Inc., CA, USA) with $488 \mathrm{~nm}$ laser excitation. Cur emission fluorescence was collected through an FITC filter. Control cells cultured without liposome transfection were used for background calibration. Kaluza software (version 1.2, Beckman Coulter) was used for data analysis.

\section{Real-time quantitative PCR assays to determine ICAM-I and E-selectin mRNA levels}

Real-time quantitative PCR assays were conducted using samples from the following experimental groups: PBS controls, C-Lipo, A-Lipo, AC-Lipo, and T-AC-Lipo. Except for the control group, HAECs were preincubated with $1 \mu \mathrm{g} / \mathrm{mL}$ LPS for 4 hours before transfection with liposomes. Each group was further divided into three subgroups with repeated treatment. The final concentration of Cur was $7.2 \mu \mathrm{g} / \mathrm{mL}$ per well. Ato concentrations were $8.0 \mu \mathrm{g} / \mathrm{mL}$ per well for the A-Lipo and AC-Lipo treatment groups and $6.0 \mu \mathrm{g} / \mathrm{mL}$ per 
well for the T-AC-Lipo group. At 48 hours post-transfection, total RNA samples were isolated from harvested cells using Trizol Reagent (Invitrogen, Karlsruhe, Germany) and used as templates for synthesis of first-strand cDNA with a PrimeScript ${ }^{\mathrm{TM}} \mathrm{RT}$ reagent Kit (Takara Biotechnology, Japan), according to the manufacturer's protocol. ICAM-1 and E-selectin mRNA expression levels were determined in triplicate using a real-time PCR system (StepOne Plus, ABI, USA) and quantified using the $2^{-\triangle \Delta \mathrm{CT}}$ method. GAPDH mRNA expression levels were determined as an internal normalization standard for each sample. Real-time PCRs were carried out in $20 \mu \mathrm{L}$ reaction mixtures (FastStart Universal SYBR Green Master, Roche Applied Science, Mannheim, Germany) containing $2 \mu \mathrm{L}$ cDNA and one pair of primers $(0.5 \mu \mathrm{M})$ for the quantification of ICAM-1, E-selectin, or GAPDH mRNA. The primer sequences were as follows: ICAM-1 forward, 5-AGCCAGTGGGCAAGAACCTT-3, reverse, 5-CGGCACGAGAAA T T GGCTCC-3; E-selectin forward, 5-TGCAAGTTCGCCTGTCCTGA-3, reverse, 5-GAGGGAGAGTCCAGCAGCAG-3; GAPDH forward, 5-CATGAGAAGTATGACAACAGCCT-3, reverse, 5-AGTCCTTCCACGATACCAAAGT-3. The programs were run using the following thermal cycling conditions: $60^{\circ} \mathrm{C}$ for 1 minute, $95^{\circ} \mathrm{C}$ for 10 minutes, then 40 cycles of $95^{\circ} \mathrm{C}$ for 15 seconds, and $58^{\circ} \mathrm{C}$ for 60 seconds.

\section{In vivo studies}

Eight-week-old ApoE knockout $\left(\mathrm{ApoE}^{-/-}\right)$mice were purchased from the Laboratory Animal Center of Sun Yat-sen University (Guangzhou, China). All surgical interventions and postoperative animal care were approved by the Institutional Animal Care and Use Committee of Sun Yatsen University, according to the Guiding Proposal for Experimental Animals issued by the Ministry of Science and Technology of China. A high-fat diet, comprising 20\% sucrose, $15 \%$ lard, $10 \%$ casein, $1.2 \%$ Chol, $0.2 \%$ sodium cholate, $0.6 \%$ calcium hydrophosphate, $0.4 \%$ stone powder, $0.4 \%$ premix, and $52.2 \%$ basal feed, was purchased from the Animal Center of Guangdong Province (Guangzhou, China). After feeding with the high-fat diet for 8 weeks, $\mathrm{ApoE}^{-/}$ knockout mice were divided into five groups: $\mathrm{ApoE}^{-/}$ model animals (control), C-Lipo, A-Lipo, AC-Lipo, and T-AC-Lipo ( $n=10$ per group). Liposome solutions $(200 \mu \mathrm{L}$, $45 \mathrm{mg} / \mathrm{kg}$ ) were injected via the tail vein. In all groups, the dosage of Cur was $2 \mathrm{mg} / \mathrm{kg}$. The dosage of Ato was $1.8 \mathrm{mg} / \mathrm{kg}$ in the A-Lipo and AC-Lipo groups and $1.4 \mathrm{mg} / \mathrm{kg}$ in the T-AC-Lipo group. Mice were injected once every 3 days for a total of 10 treatments. Wild-type (WT) C57BL/6 mice were used as a negative control group. Mice in all groups were fed with the high-fat diet throughout the treatment course and sacrificed 30 days after first dose of liposomes was applied. Mouse plasma samples were collected to evaluate the concentration of HMG-CoA reductase and lipid levels, including serum total cholesterol (TC), TG, and low-density lipoprotein cholesterol (LDL-C). Aortas extracted from animals were split and peeled under an SZM45T1 stereomicroscope (SEEPACK, Shenzhen, China) as reported. ${ }^{42,43}$ Aorta samples were immersed in 4\% paraformaldehyde for 5 minutes and then washed in $60 \%$ fresh isopropanol for 1 minute. After staining in $60 \%$ Oil-Red-O solution, aortas were washed three times with PBS to remove the excess Oil-Red-O. Finally, samples were mounted on glass slides and imaged using a D550 digital camera (Cannon, Tokyo, Japan). Sections of mouse hearts and aortic root lesions were subjected to $\mathrm{H} \& \mathrm{E}$ and immunofluorescence staining. Frozen sections were sequentially incubated with ICAM-1 and E-selectin monoclonal antibodies (Abcam Co., Ltd., Cambridge, UK). After washing with PBS three times, sections were further incubated with AF555-(E-selectin-stained samples) and AF647-(ICAM stained samples) labeled secondary antibodies. Nuclei were stained with DAPI. AF555 and AF647 fluorescence (excitation 552 and $638 \mathrm{~nm}$; emission 570 and $665 \mathrm{~nm}$, respectively) were detected by CLSM to evaluate the E-selectin and ICAM-1 protein levels. Moreover, IL-6 and MCP-1, inflammatory cytokines secreted by plaques, were evaluated by immunofluorescence assay. AF555- and AF647labeled secondary antibodies (Abcam Co., Ltd., Cambridge, UK) were used to detect IL-6 and MCP-1, respectively.

\section{Statistical analysis}

Data are expressed as mean $\pm \mathrm{SD}$. Data were analyzed by one-way ANOVA (SPSS software, version 19.0, SPSS Inc.) to calculate $P$-values. $P$-values $<0.05$ were considered significant.

\section{Results \\ Preparation and characterization of the targeting-ligand-modified liposomes}

The method of preparation of Ato and Cur-encapsulated liposomes (AC-Lipo) is outlined in Figure 1. Specific amounts of Ato and Cur were added to a lipid mixture containing DPPC, Chol, and PEG-modified DSPE (ratio, 5:1:1) in chloroform to generate AC-Lipo, according to a previously reported technique. ${ }^{27}$ The shape and size distributions of liposomes were investigated using TEM and DLS. Regardless of whether or not Cur and Ato were loaded, liposomes 

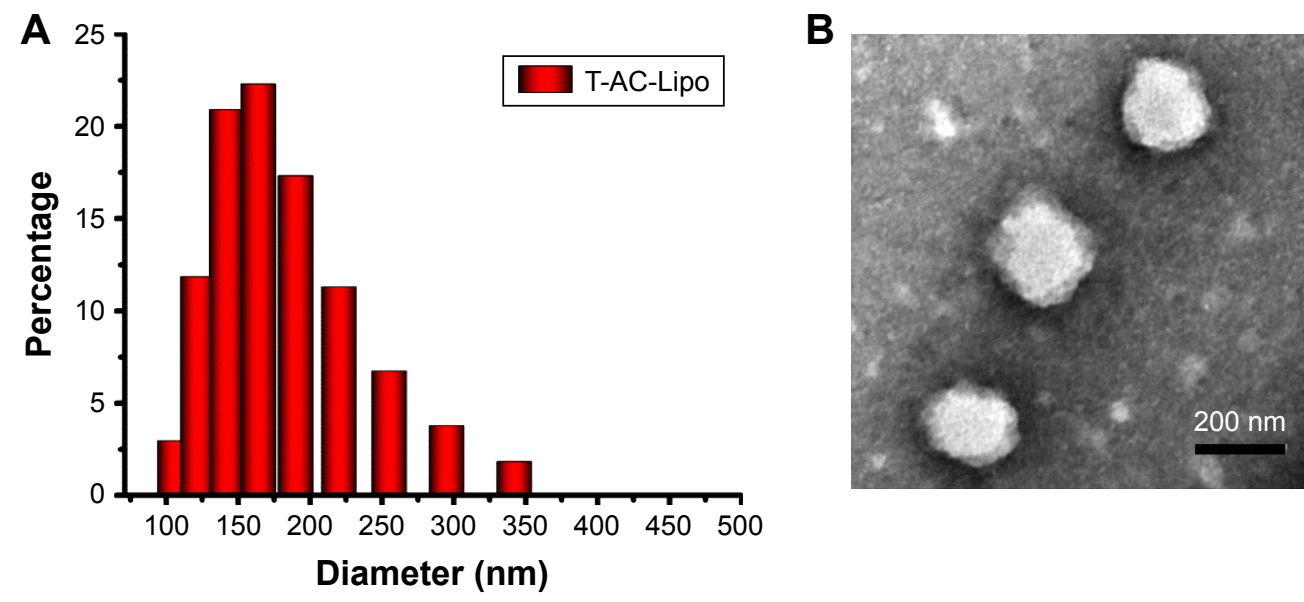

Figure 2 Particle size (A) and transmission electron microscopic image (B) of the targeting liposomes encapsulating atorvastatin calcium and curcumin (T-AC-Lipo).

exhibited spherical morphology with an average diameter of approximately $190 \mathrm{~nm}$, indicating that encapsulation of Cur and/or Ato had no significant influence on their size or morphology (Figure 2 and Table 1). Encapsulation of Ato and Cur decreased the negative charge of liposomes by different amounts. Compared to B-Lipo (control liposomes without Ato or Cur), the zeta potentials of C-Lipo and A-Lipo decreased from $-13.9 \pm 0.61$ to $-3.69 \pm 0.60$ and $-8.74 \pm 0.58 \mathrm{mV}$, respectively, owing to the positive charges of Ato and Cur (Table 1). AC-Lipo had a moderate negative zeta potential of $-6.94 \pm 0.48 \mathrm{mV}$. In addition, co-encapsulation of Cur had no significant effect on the DLC or DLE of Ato. As shown in Table 1, DLC and DLE values for AC-Lipo were almost the same as those for A-Lipo $(4.01 \% \pm 0.05 \%$ vs $3.82 \% \pm 0.05 \%$ and $87.48 \% \pm 3.27 \%$ vs $84.16 \% \pm 4.25 \%$, respectively).

As negative charges can hinder cell uptake of liposomes, the targeting ligand, Esb peptide, was introduced to the liposome surface to enhance drug delivery efficiency. Hence, Esb peptide-modified PEG-DSPE (Esb-PEG-DSPE) was synthesized and used in place of PEG-DSPE for the preparation of targeting liposomes (T-AC-Lipo), which specifically bind E-selectin on the membranes of inflamed ECs. ${ }^{44}$ Additionally,
Esb peptide modification had no significant impact on liposome size, zeta potential, DLC, or DLE (Table 1).

\section{Cytotoxicity}

As shown in Figure 3A, even at high concentration ( $200 \mu \mathrm{g} / \mathrm{mL}$ liposome and $8.8 \mu \mathrm{g} / \mathrm{mL}$ Cur), the viabilities of cells treated with B-Lipo and C-Lipo were $\sim 80 \%$, indicating that both liposomes and Cur exhibited minor cytotoxicity to HAECs. Nevertheless, treatment with A-Lipo containing $8.0 \mu \mathrm{g} / \mathrm{mL}$ Ato reduced cell viability to $55.72 \%$. In contrast, treatment with a combination of Ato and Cur (AC-Lipo) resulted in cell viability of $63.84 \%$, while treatment with targeting liposomes (T-AC-Lipo) further enhanced cell viability to $70.12 \%$ (Figure $3 \mathrm{~B}$ ).

\section{Transfection efficiency}

The transfection efficiencies of liposomes were evaluated using CLSM and flow cytometry. HAECs in the nontargeting (AC-Lipo), targeting (T-AC-Lipo), and competitive (T-ACLipo plus preincubation with Esb peptide) liposome groups were imaged at 4 and 12 hours post-transfection. As shown in Figure 4A, Cur fluorescence (green) increased in all groups

Table I Particle size, zeta potential, and curcumin and atorvastatin calcium loading content and efficiency of B-Lipo, C-Lipo, A-Lipo, AC-Lipo, and T-AC-Lipo

\begin{tabular}{l|l|l|l|l|l|l}
\hline & $\begin{array}{l}\text { Size } \\
(\mathbf{n m})\end{array}$ & $\begin{array}{l}\text { Zeta potential } \\
(\mathbf{m V})\end{array}$ & $\begin{array}{l}\text { Cur loading } \\
\text { content }(\%)\end{array}$ & $\begin{array}{l}\text { Ato loading } \\
\text { content }(\%)\end{array}$ & $\begin{array}{l}\text { Cur loading } \\
\text { efficiency }(\%)\end{array}$ & $\begin{array}{l}\text { Ato loading } \\
\text { efficiency (\%) }\end{array}$ \\
\hline B-Lipo & $190 \pm 5.0$ & $-13.9 \pm 0.61$ & - & - & - & - \\
C-Lipo & $178 \pm 3.3$ & $-3.69 \pm 0.60$ & $4.39 \pm 0.01$ & - & $86.12 \pm 4.28$ & - \\
A-Lipo & $182 \pm 4.3$ & $-8.74 \pm 0.58$ & - & $4.01 \pm 0.05$ & - & $87.48 \pm 3.27$ \\
AC-Lipo & $180 \pm 5.4$ & $-6.94 \pm 0.48$ & $3.45 \pm 0.02$ & $3.82 \pm 0.05$ & $68.58 \pm 2.16$ & $84.16 \pm 4.25$ \\
T-AC-Lipo & $192 \pm 0.8$ & $-6.78 \pm 0.99$ & $4.00 \pm 0.07$ & $3.36 \pm 0.01$ & $79.33 \pm 3.61$ & $74.55 \pm I .70$ \\
\hline
\end{tabular}

Notes: Size was determined by DLS. Data are presented as mean \pm SD $(n=3)$.

Abbreviations: A-Lipo, atorvastatin calcium-loaded liposome; AC-Lipo, atorvastatin calcium- and curcumin-loaded liposome; Ato, atorvastatin calcium; B-Lipo, blank liposome; Cur, curcumin; C-Lipo, curcumin-loaded liposome; T-AC-Lipo, targeting-ligand-modified AC-Lipo. 
A

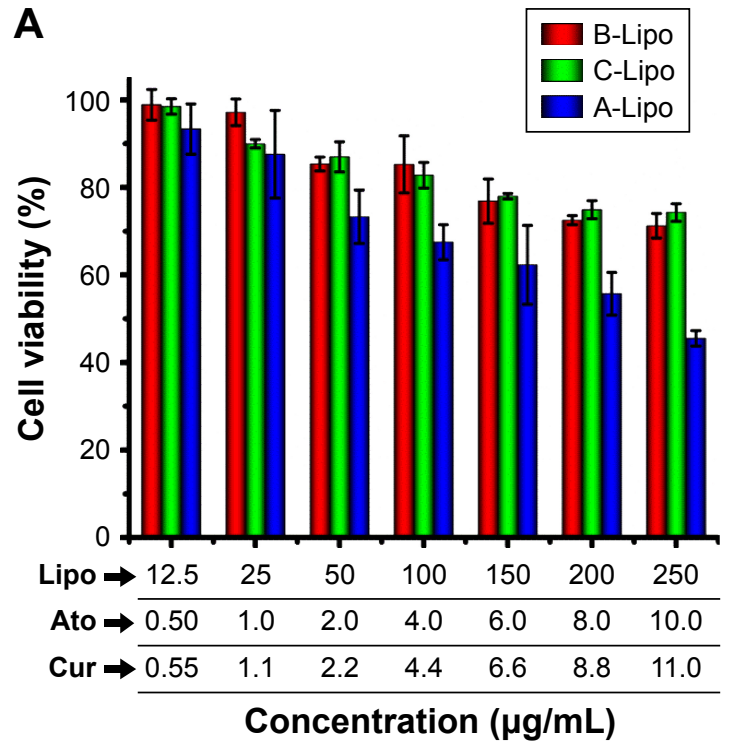

B
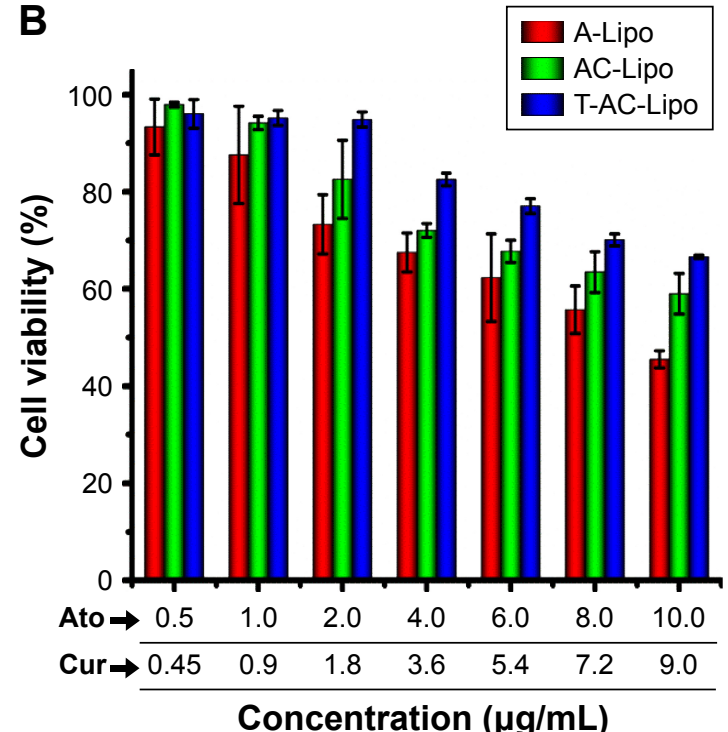

Figure 3 (A) HAEC viability following treatment with B-Lipo, C-Lipo, and A-Lipo at various concentrations of liposomes, Ato, and Cur. The Cur and Ato loading content were $4.39 \%$ and $4.01 \%$, respectively. (B) HAEC viability following treatment with A-Lipo, AC-Lipo, and T-AC-Lipo. Data were generated by MTT assay (mean \pm SE; $n=3$ ). Abbreviations: A-Lipo, atorvastatin calcium-loaded liposome; Ato, atorvastatin calcium; B-Lipo, blank liposome; C-Lipo, Cur-loaded liposome; Cur, curcumin; AC-Lipo, atorvastatin calcium- and curcumin-loaded liposome; HAEC, human aortic endothelial cell; T-AC-Lipo, targeting-ligand (Esb peptide)-modified atorvastatin calcium- and curcumin-loaded liposome.

over time, with the highest levels in cells treated with T-ACLipo at 12 hours post-transfection. Cells preincubated with $1 \mu \mathrm{g} / \mathrm{mL}$ Esb peptide then treated with T-AC-Lipo exhibited similar green fluorescence intensity to those treated with AC-Lipo at 12 hours post-transfection, indicating that cell uptake of T-AC-Lipo was inhibited in the presence of excess Esb peptide. The results of flow cytometry were consistent with those of CLSM. As shown in Figure 4B, the percentage of Cur fluorescence-positive cells in the T-AC-Lipo group (99.77\%) was approximately 5.17 times that of the nontargeting (AC-Lipo) group (19.29\%). Preincubation with Esb peptide clearly reduced transfection efficiency (Figure 4B). Moreover, the fluorescence intensity of cells in the targeting group was approximately 15 times that of the nontargeting

A
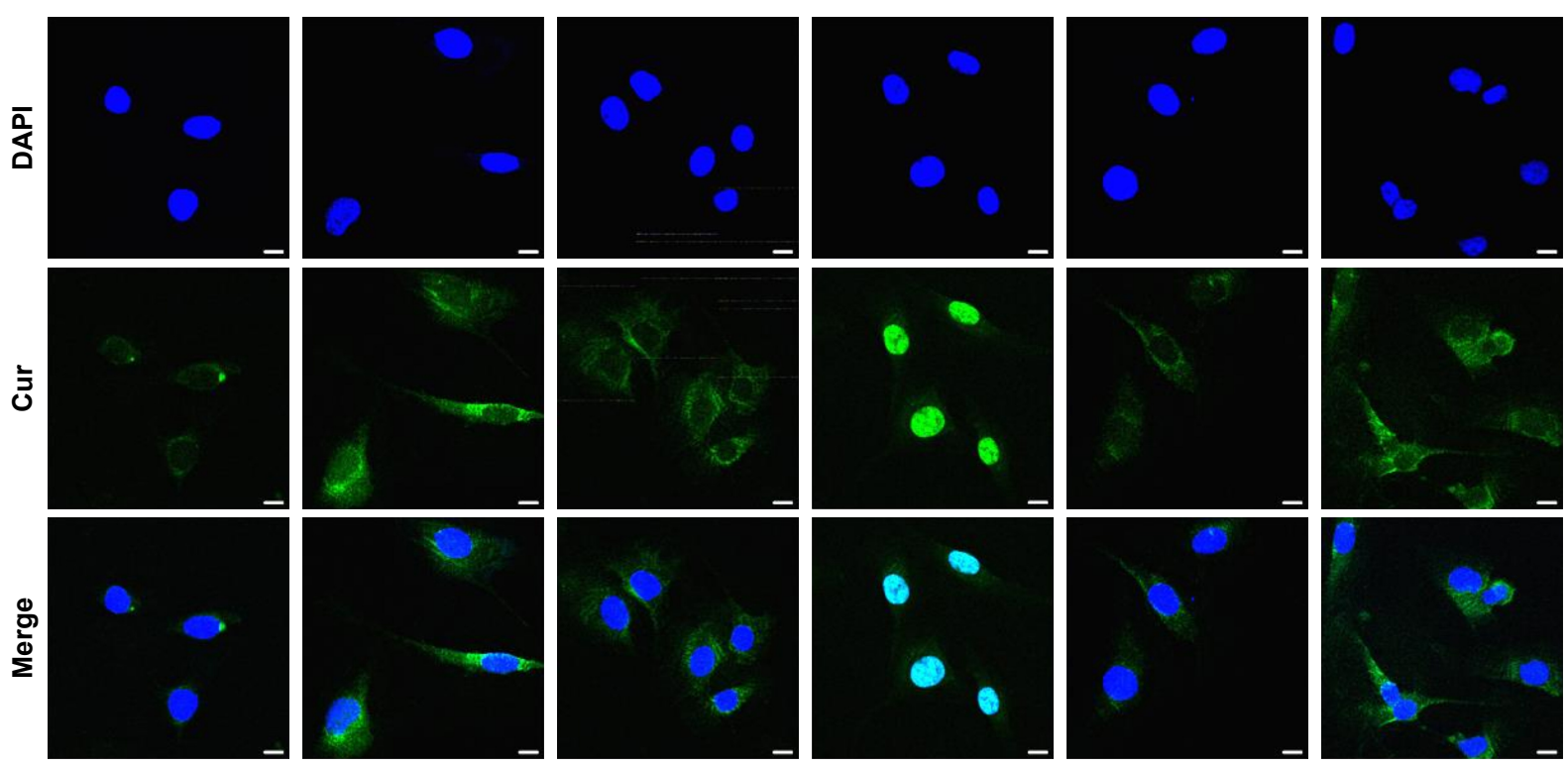

4 hours

12 hours

AC-Lipo

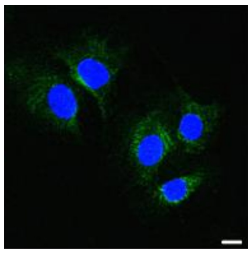

4 hours

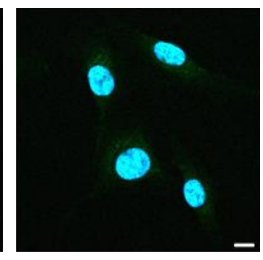

12 hours

T-AC-Lipo

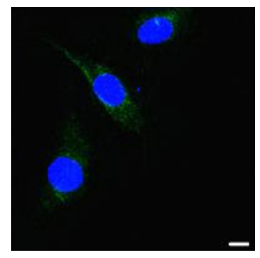

4 hours

T-AC-Lipo+T

Figure 4 (Continued) 


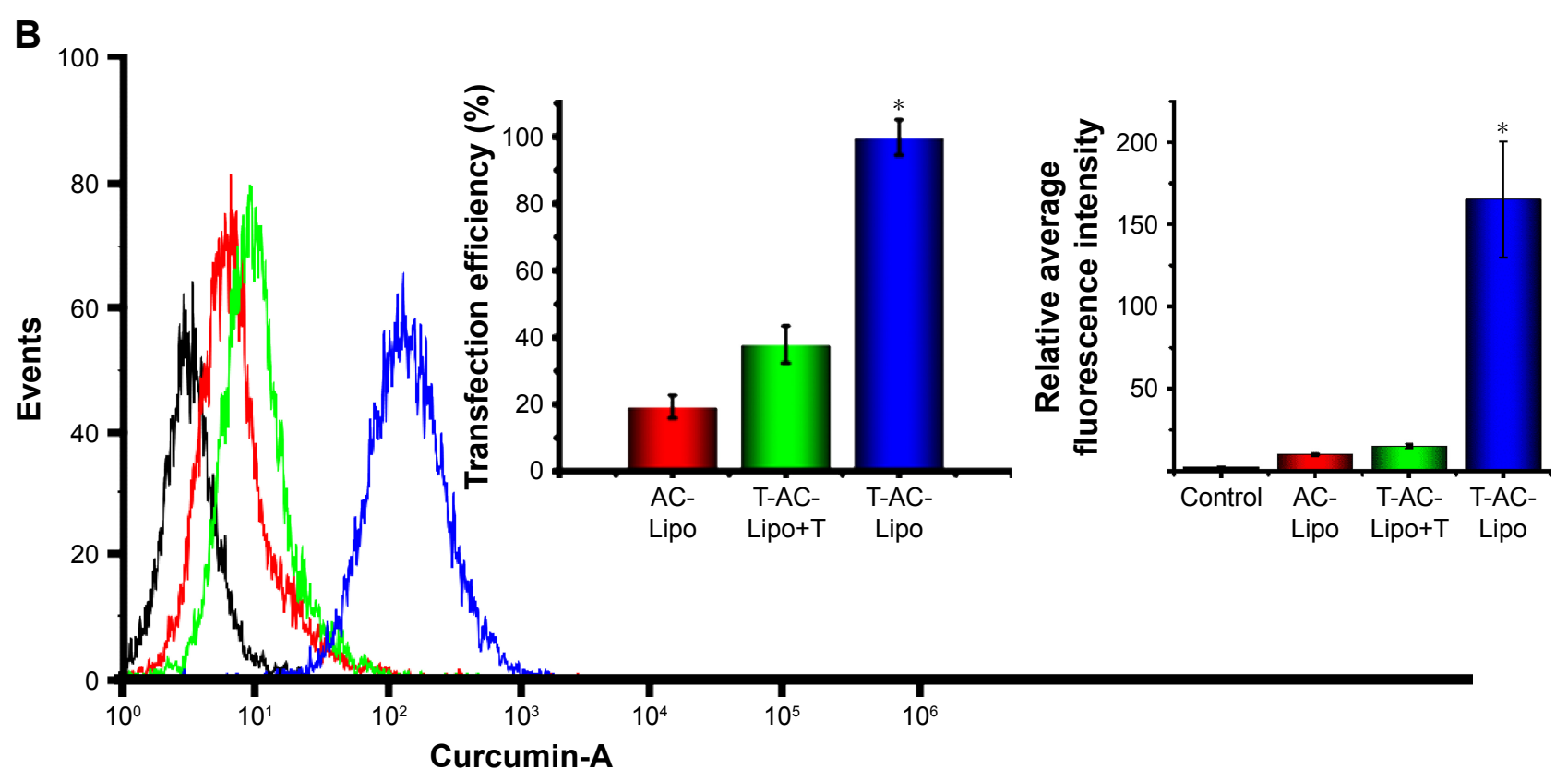

Control $=$ AC-Lipo $=$ T-AC-Lipo+T T-AC-Lipo

Figure 4 (A) Confocal laser microscopic images of human aortic endothelial cells incubated with AC-Lipo (nontargeted group), T-AC-Lipo (targeted group), and T-ACLipo+T (competitive inhibition group, preincubated with I $\mu \mathrm{g} / \mathrm{mL}$ targeting peptide Esb before treatment with T-AC-Lipo). Nuclei were stained with DAPI (blue fluorescence). Cur emits green fluorescence. Scale bars, $10 \mu \mathrm{m}$. (B) Quantitative analysis of transfection efficiency by flow cytometry. The statistical significance was analyzed using the Student's $t$-test. Data are presented as mean $\pm S D(n=3),{ }^{*} P<0.05$. Cur concentration was $7.2 \mu g / \mathrm{mL}$ per well. Before transfection with various liposomes, cells were preincubated with I $\mu \mathrm{g} / \mathrm{mL}$ lipopolysaccharide for 4 hours.

Abbreviations: AC-Lipo, atorvastatin calcium- and curcumin-loaded liposome; Cur, curcumin; T-AC-Lipo, targeting-ligand-modified atorvastatin calcium- and curcuminloaded liposome.

and competitive groups. These results demonstrate that modification with Esb peptide significantly enhanced liposome transfection efficiency.

\section{The synergistic effect of Ato and Cur on the expression of inflammatory factors}

The initiation and development of atherosclerosis are closely associated with the inflammation at the lesion site. The dysfunctional endothelium secretes adhesion molecules (eg, E-selectin and ICAM-1), which attract inflammatory cells and trigger inflammation in the atherosclerotic lesion. ${ }^{45}$ LPS can be used to induce overexpression of E-selectin and ICAM-1 in ECs by inducing an inflammatory reaction. ${ }^{46}$ As shown in Figure S1, E-selectin and ICAM-1 mRNA levels were remarkably upregulated by LPS $(1 \mu \mathrm{g} / \mathrm{mL})$ over time, reaching a maximum 4 hours after LPS addition. Compared with control cells not treated with LPS, relative E-selectin and ICAM-1 mRNA levels increased by 76.66 and 52.17 times, respectively, 4 hours after LPS stimulation. Hence, preincubation with LPS for 4 hours was used to upregulate expression of E-selectin and ICAM-1. C-Lipo treatment slightly suppressed LPS-induced E-selectin and ICAM-1 gene expression. In contrast, A-Lipo treatment significantly inhibited E-selectin and ICAM-1 expression by $54.15 \%$ and $28.62 \%$, respectively, as compared to levels in LPS-pretreated cells (Figure S1). Cells in the AC-Lipo treatment group expressed lower E-selectin and ICAM-1 mRNA levels than those in the A-Lipo group. Moreover, Esb peptide (T-AC-Lipo) further enhanced the inhibition effect of combined therapy on E-selectin and ICAM-1 mRNA levels by $59.38 \%$ and $34.42 \%$, respectively (Figure 5A). These results demonstrate that targeting combination treatment (T-AC-Lipo) achieved far superior inhibition of E-selectin and ICAM-1 gene expression than either single-drug treatment (A-Lipo or C-Lipo) or nontargeting combination treatment (AC-Lipo). To investigate the suppression effects of Ato and Cur at the protein level, immunofluorescent staining of E-selectin and ICAM-1 proteins was performed in HAECs; E-selectin protein, ICAM-1 protein, and the nucleus were stained yellow, purple, and blue, respectively. As shown in Figure 5B, C-Lipo treatment resulted in relatively weak yellow (E-selectin) and purple (ICAM-1) fluorescence, while A-Lipo treatment significantly reduced both E-selectin and ICAM-1 fluorescence. The weakest E-selectin and ICAM-1 staining was observed in cells of the T-AC-Lipo treatment group. 
The results of immunofluorescent staining were consistent with those determined by real-time quantitative PCR and further demonstrated that EC-targeted liposomes incorporating Ato and Cur (T-AC-Lipo) could remarkably inhibit E-selectin and ICAM-1 levels.

Moreover, we evaluated ROS levels in HAECs treated with each of the different liposome preparations by flow cytometry. As shown in Figure 5C, HAECs produced abundant ROS following treatment with LPS, indicating that LPS-activated dysfunctional ECs overexpressed ROS. ROS levels in the C-Lipo treatment were significantly reduced owing to the superior antioxidative properties of Cur. However, the antioxidative properties of Ato were limited. Notably, the T-AC-Lipo treatment exhibited the greatest antioxidative effects due to the ability of Esb peptide to strongly target dysfunctional ECs.
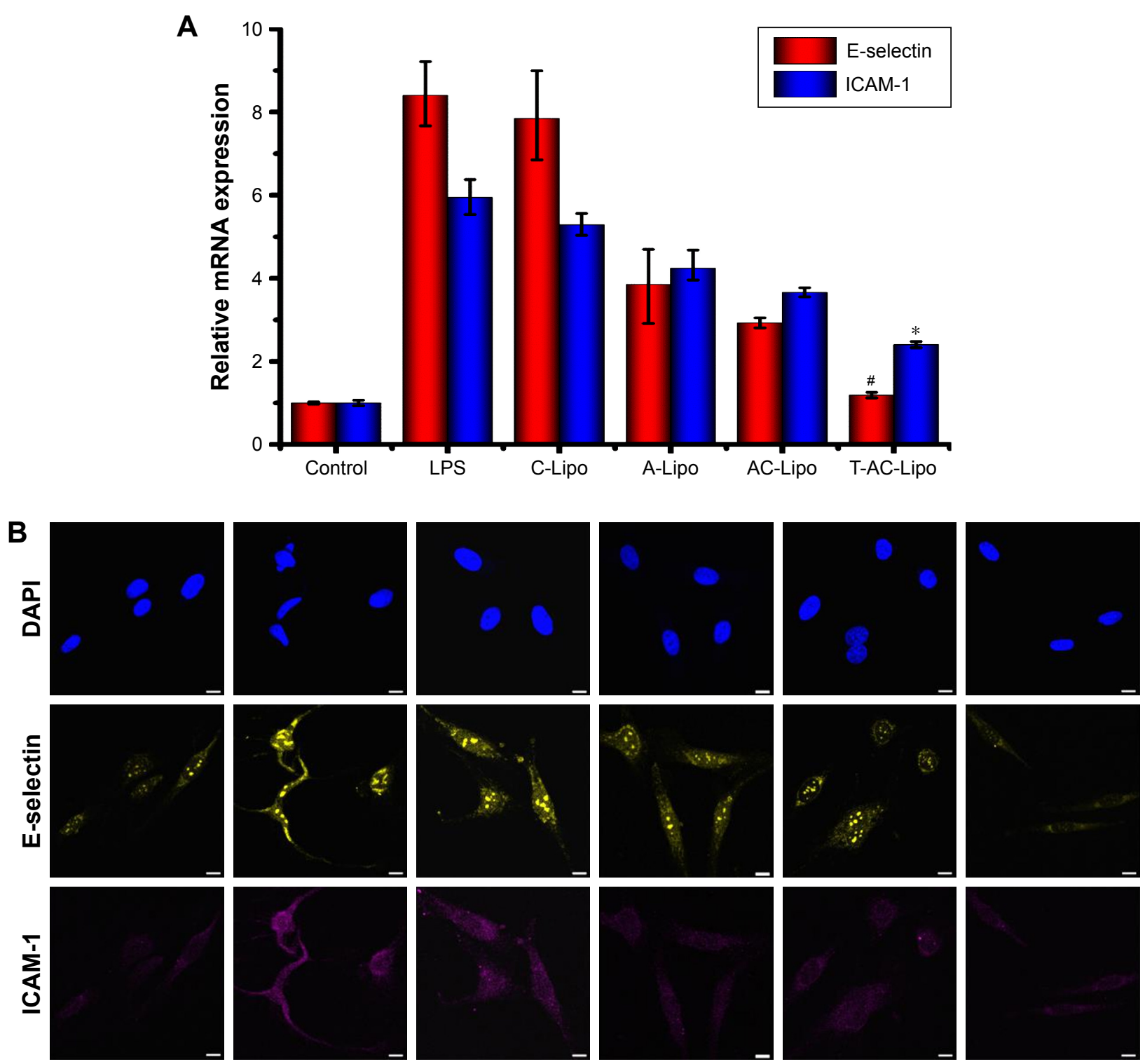

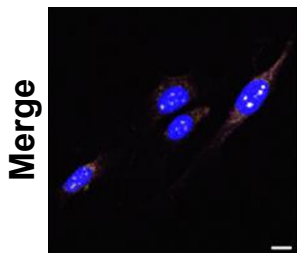

Control

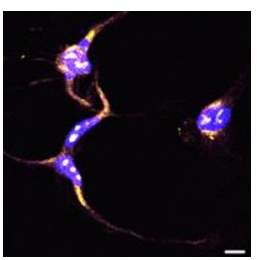

LPS

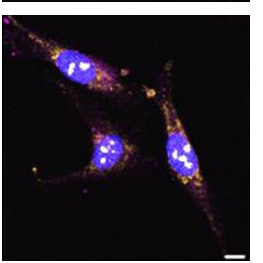

C-Lipo

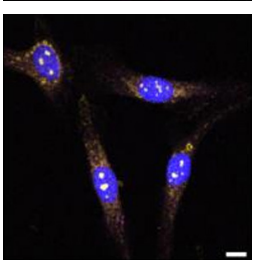

A-Lipo

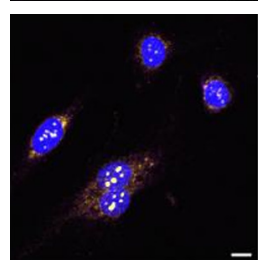

AC-Lipo

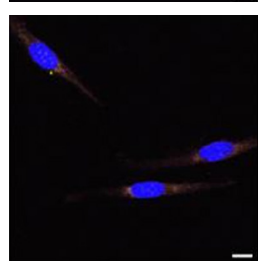

T-AC-Lipo

Figure 5 (Continued) 

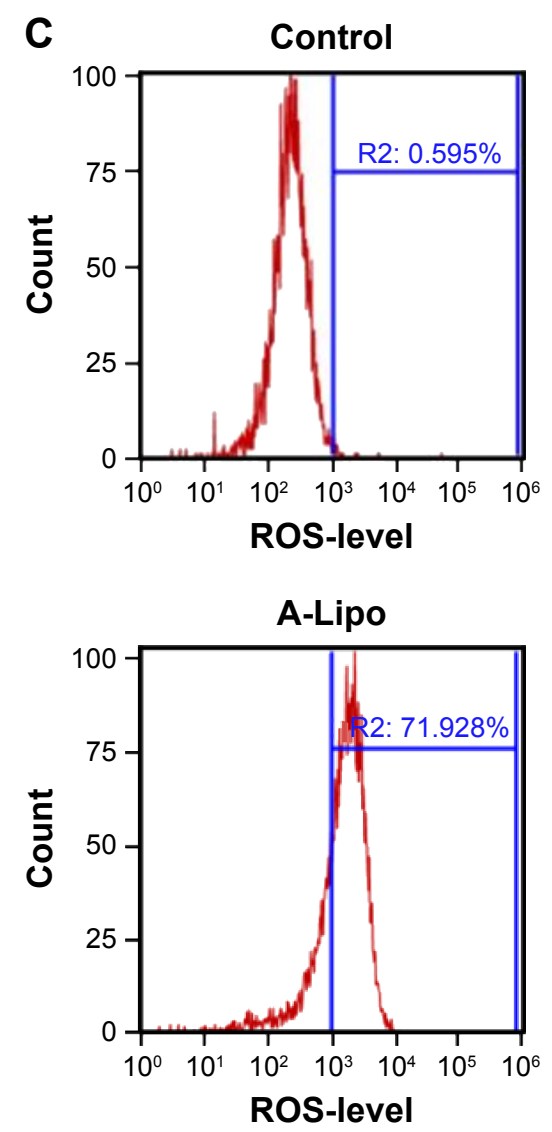
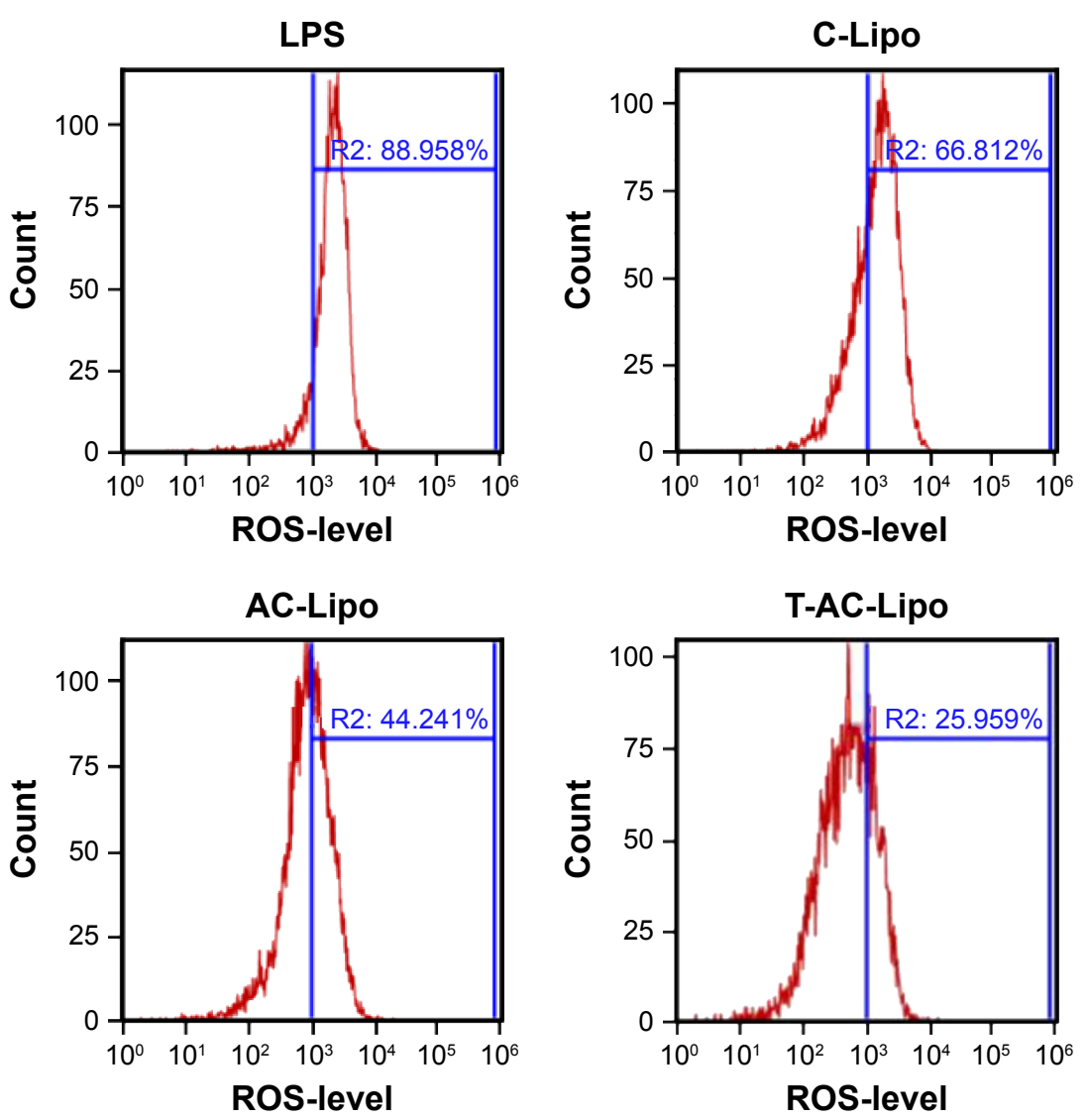

Figure 5 (A) The synergistic effect of Ato and Cur on E-selectin and ICAM-I gene expression in HAECs determined by real-time quantitative PCR (mean \pm SD; $n=3$; $* P<0.05$ and ${ }^{\# P}<0.05$, compared to AC-Lipo). After preincubation with I $\mu \mathrm{g} / \mathrm{mL}$ LPS for 4 hours, cells were transfected with various liposomes and then harvested to evaluate gene expression 24 hours after transfection. (B) Evaluation of E-selectin and ICAM-I protein expression in HAECs treated with various liposomes by immunofluorescence assay. Yellow and pink fluorescence represent E-selectin and ICAM-I proteins, respectively. Scale bars, $10 \mu \mathrm{m}$. (C) ROS levels in HAECs treated with various liposome medicines determined by flow cytometry. Liposomes were used at $200 \mu \mathrm{g} / \mathrm{mL}$, where applied. Ato was used at $8.0 \mu \mathrm{g} / \mathrm{mL}$ in cells treated with A-Lipo and AC-Lipo and at $6.0 \mu \mathrm{g} / \mathrm{mL}$ in T-ACLipo-treated cells. Cur was used at $7.2 \mu \mathrm{g} / \mathrm{mL}$ for cells treated with A-Lipo, AC-Lipo, or T-AC-Lipo. All groups except controls were pretreated with I $\mu$ g/mL LPS for 4 hours. Abbreviations: A-Lipo, atorvastatin calcium-loaded liposome; AC-Lipo, atorvastatin calcium- and curcumin-loaded liposome; HAEC, human aortic endothelial cell; ICAM-I, intercellular cell adhesion molecule-I; LPS, lipopolysaccharide; ROS, reactive oxygen species; T-AC-Lipo, targeting-ligand-modified atorvastatin calcium- and curcuminloaded liposome.

\section{In vivo studies}

To assess their anti-atherosclerotic effects, various liposomes were administrated to $\mathrm{ApoE}^{-/-}$mice via intravenous injection in the tail. ${ }^{47}$ Atherosclerotic lesions were quantified by staining lipid red with Oil-Red-O. ${ }^{22,43}$ As shown in Figure $6 \mathrm{~A}$ and $\mathrm{C}$, in the control group (untreated $\mathrm{ApoE}^{-/}$ mice), $61.58 \%$ of the thoracic aorta was covered with red plaques, while treatment with A-Lipo and C-Lipo reduced the lesion areas to $39.69 \%$ and $56.57 \%$, respectively. There was no significant difference between lesion areas in the A-Lipo and AC-Lipo treatment groups (39.69\% vs $31.52 \%$ ), maybe due to low levels of cell uptake. In contrast, the percentage lesion area in the thoracic aorta of animals receiving T-ACLipo was reduced to $17.14 \%$.

The severity of atherosclerotic lesions was further assessed by H\&E staining of cross sections of the aortic root (Figure 6B-D). Typical symptoms of atherosclerotic lesions (ie, foam cell infiltration and extensive Chol clefts) were evident in sections from control group animals. In contrast, sections from mice treated with T-AC-Lipo presented few foam cells and grossly normal intimal structures. As shown in Figure 6D, the average lesion area in sections from control group animals was $0.1409 \mathrm{~mm}^{2}$, while treatment with A-Lipo or C-Lipo resulted in a $51.59 \%$ or $32.43 \%$ reduction to 0.0682 or $0.0952 \mathrm{~mm}^{2}$, respectively. Targeted treatment with T-AC-Lipo further decreased the lesion area by $76.58 \%$ to $0.0330 \% \mathrm{~mm}^{2}$ relative to the control group. These results provide evidence of the enhanced anti-atherosclerosis effects achieved by using a combination of Ato and Cur delivered by EC-targeted liposomes.

Immunofluorescent staining of tissue samples and evaluation of plasma lipid levels were conducted to further elucidate the potential anti-atherosclerotic mechanism underlying the effects of combined treatment with Ato and Cur. 
A
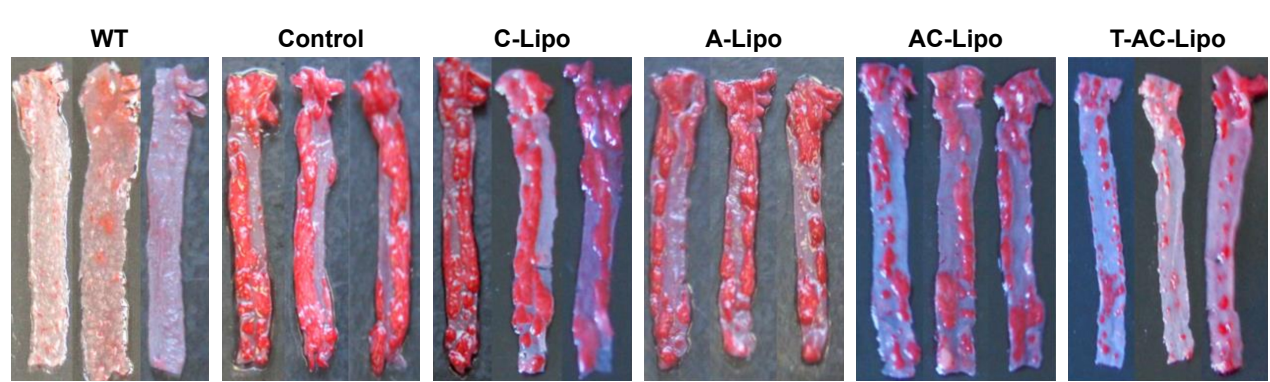

B
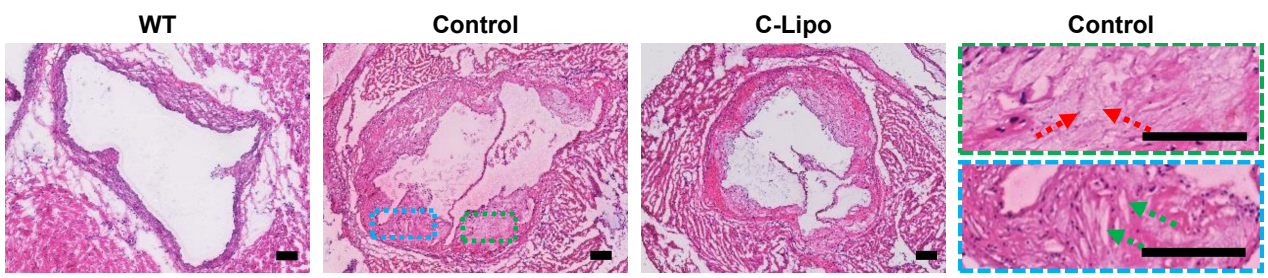

T-AC-Lipo

T-AC-Lipo
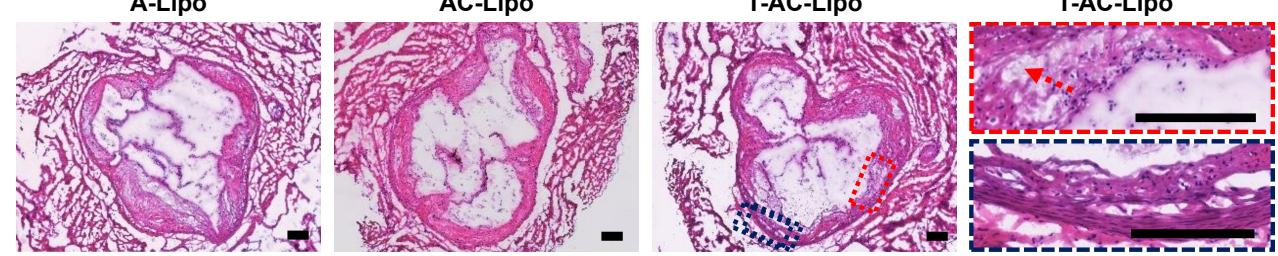

C
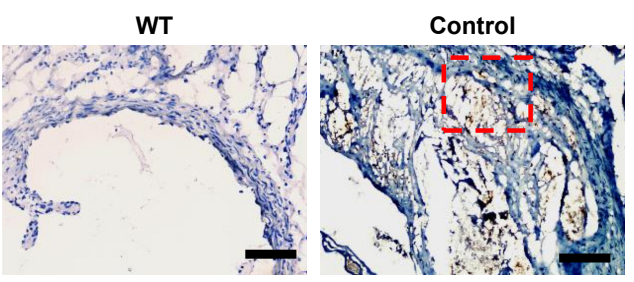

C-Lipo

Control
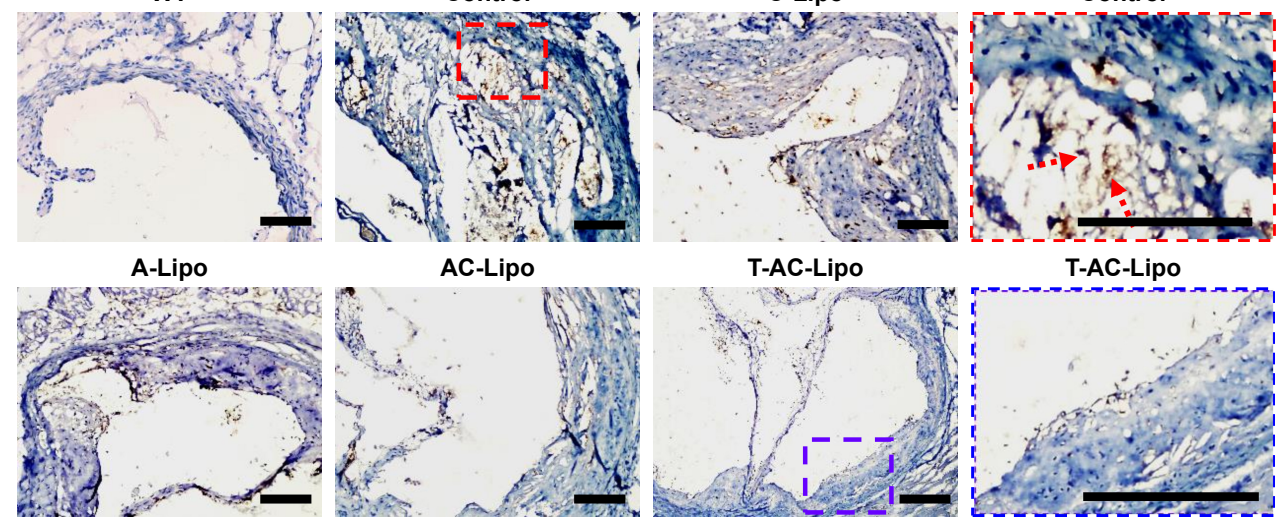

T-AC-Lipo

T-AC-Lipo

D
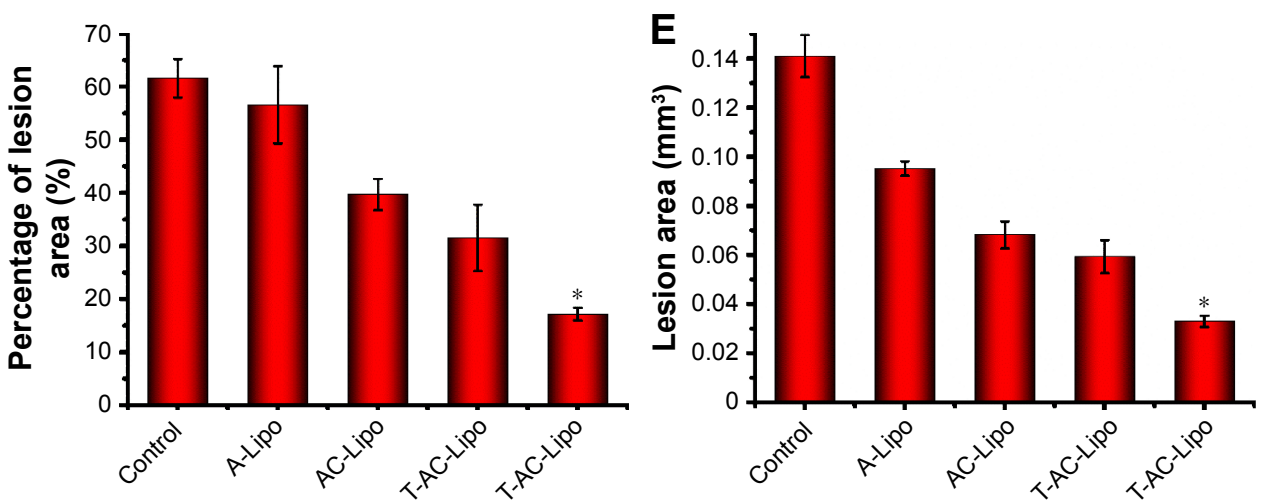

Figure 6 Oil-Red-O-stained thoracic aorta (A) and H\&E-stained aortic root lesion (B) images for histologic analysis of atherosclerosis in ApoE knockout mice 30 days after treatment with various liposomes. Lipids in atherosclerotic lesions were stained red with Oil-Red-O in (A). The dashed green and blue rectangular areas in the control group section (ApoE knockout mouse) and the dashed red and dark blue rectangular areas in the T-AC-Lipo treatment group section are magnified in (B). The dashed red and green arrows indicate foam cells and cholesterol clefts, respectively. Infiltrating macrophages in atherosclerotic lesions were labeled with Mac-3 antibody by immunohistochemistry staining (C). Red dashed arrows indicate macrophages in the plaques. (D) and (E) Quantitative analysis of the areas of red atherosclerotic lesions in Figure 6A ( $n=3$ ) and the cross-sectional areas of atherosclerotic lesions in Figure 6B $(n=3)$, respectively. $* P<0.05$ compared to other treatments. WT (C57BL/6) mouse group. Control group, atherosclerotic mouse model (ApoE knockout mouse). Scale bars, $50 \mu \mathrm{m}$. Liposome concentration, $45 \mathrm{mg} / \mathrm{kg}$ body weight. Cur concentration, $2 \mathrm{mg} / \mathrm{kg}$ body weight. Ato concentration for A-Lipo and AC-Lipo treatment groups, $1.8 \mathrm{mg} / \mathrm{kg}$ body weight. Ato concentration for T-AC-Lipo treatment group, $1.4 \mathrm{mg} / \mathrm{kg}$ body weight.

Abbreviations: A-Lipo, atorvastatin calcium-loaded liposome; AC-Lipo, atorvastatin calcium- and curcumin-loaded liposome; T-AC-Lipo, targeting-ligand-modified atorvastatin calcium- and curcumin-loaded liposome; WT, wild type. 
As shown in Figure 7, sections from animals in the A-Lipo group showed weaker fluorescent staining of E-selectin and ICAM-1 proteins relative to the B-Lipo (control) group. Combined treatment with Cur and Ato (AC-Lipo) led to a further decrease of E-selectin and ICAM-1 levels, while treatment with T-AC-Lipo led to the lowest staining intensities of these proteins.

Inflammatory factors (eg, IL-6) and chemokines (eg, MCP-1) are closely associated with the development and progression of atherosclerosis. ${ }^{2,48}$ For example, IL-6 can activate the nuclear factor kappa B to promote macrophage infiltration and induce ECs release of MCP-1, leading to elevated recruitment of monocytes and lymphocytes. ${ }^{49}$ Therefore, the expression levels of IL-6 and MCP-1 were also evaluated (Figure 8). Sections from animals treated with T-AC-Lipo showed much weaker fluorescence staining of IL-6 and MCP-1 than those receiving A-Lipo or C-Lipo alone. These results indicate that combined treatment with T-AC-Lipo effectively reduces atherosclerosis by suppressing inflammatory factors at both the mRNA and protein levels, thereby inhibiting macrophage infiltration of the arterial intima.

In addition, A-Lipo and C-Lipo treatments decreased TC, TG, and LDL-C levels by inhibiting HMG-CoA reductase (Table 2). Animals receiving T-AC-Lipo treatment had the lowest plasma TC, TG, and LDL-C levels, due to the decreased activity of HMG-CoA reductase. The plasma TC contents of animals receiving A-Lipo and C-Lipo were $59.41 \%$ and $80.67 \%$ relative to that of untreated animals (control group). Compared to A-Lipo and C-Lipo treatment alone, T-AC-Lipo treatment reduced TC content by $35.95 \%$ and $52.83 \%$ and LDL-C by $38.25 \%$ and $52.73 \%$, respectively (Table 2 ). Taken together, our data demonstrate that a combination of Ato and Cur can synergistically reduce atherosclerosis via decreasing lipid deposition, ${ }^{6,50}$ and the expression of inflammation factors ${ }^{2,48}$ in atherosclerotic lesions.
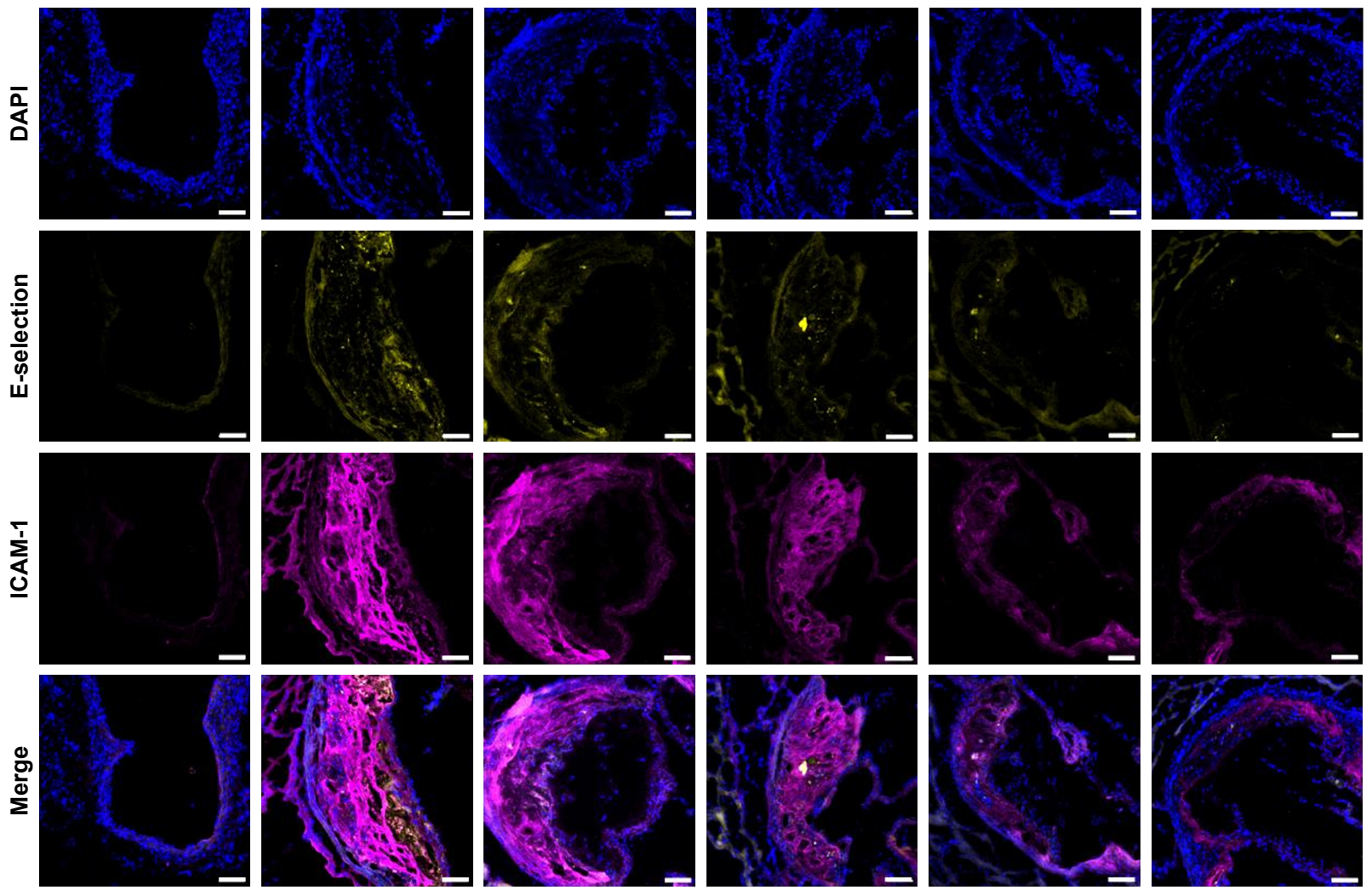

WT

Control

C-Lipo

A-Lipo

AC-Lipo

T-AC-Lipo

\section{ApoE ${ }^{-/-}$mice}

Figure 7 Expression of E-selectin and ICAM-I proteins in aortic root lesions determined by immunofluorescence analysis.

Notes: Yellow and pink fluorescence represent E-selectin and ICAM-I proteins, respectively. WT, wild-type mouse (C57BL/6) group. Control group, atherosclerotic mouse model (ApoE knockout mouse, ApoE ${ }^{-}-$). Scale bars, $100 \mu \mathrm{m}$. Liposome concentration, $45 \mathrm{mg} / \mathrm{kg}$ body weight. Cur concentration, $2 \mathrm{mg} / \mathrm{kg}$ body weight. Ato concentration for A-Lipo and AC-Lipo treatment groups, $1.8 \mathrm{mg} / \mathrm{kg}$ body weight. Ato concentration for the T-AC-Lipo treatment group, $1.4 \mathrm{mg} / \mathrm{kg}$ body weight.

Abbreviations: A-Lipo, atorvastatin calcium-loaded liposome; AC-Lipo, atorvastatin calcium- and curcumin-loaded liposome; Ato, atorvastatin calcium; Cur, curcumin; ICAM-I, intercellular cell adhesion molecule-I; T-AC-Lipo, targeting-ligand-modified atorvastatin calcium- and curcumin-loaded liposome. 

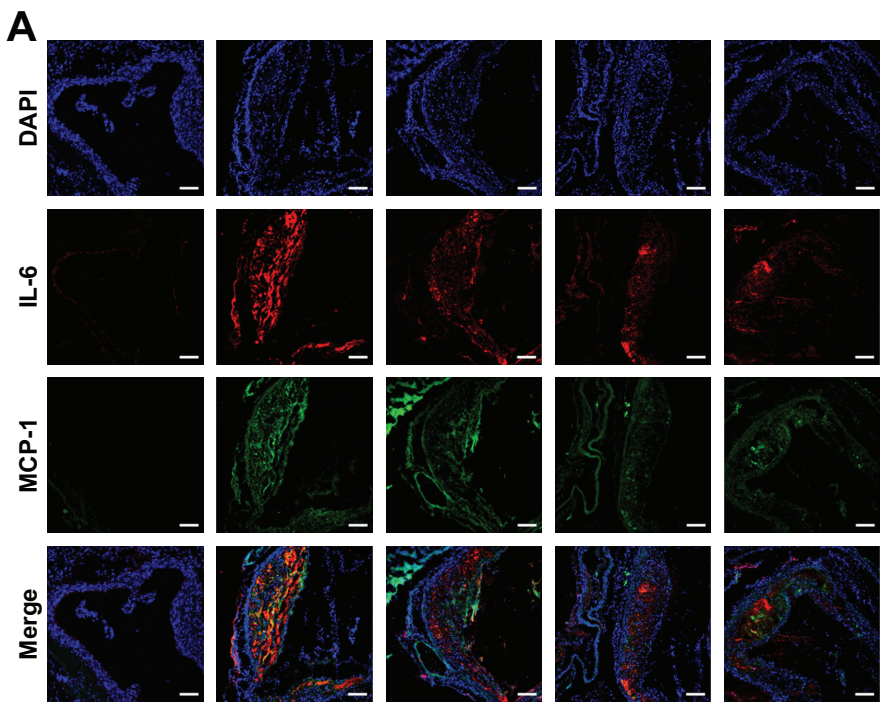

Control
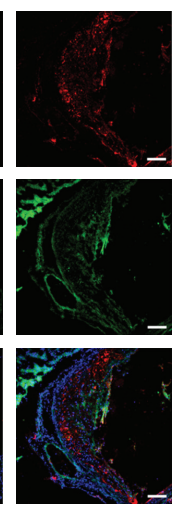

C-Lipo
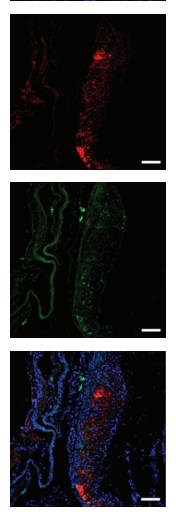

A-Lipo
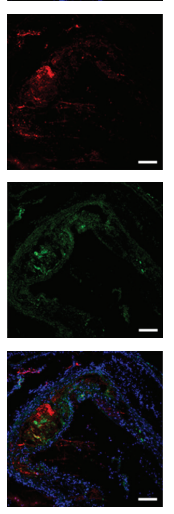

AC-Lipo
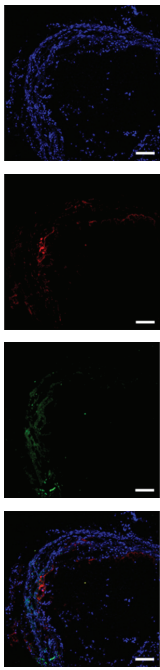

T-AC-Lipo
B

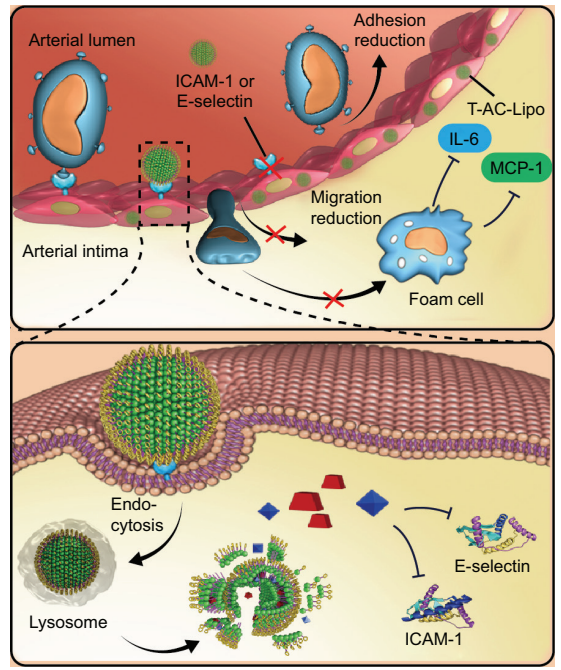

ApoE ${ }^{-/-}$mice

Figure 8 (A) Expression of IL-6 and MCP-I proteins in aortic root lesions determined by immunofluorescence analysis. Red and green fluorescence represent IL-6 and MCP-I, respectively. WT mice (C57BL/6). Control group and various liposome treatment groups comprised atherosclerotic model $\left(A p o E^{-1-}\right)$ mice. Scale bars, I00 $\mu \mathrm{m}$. Liposome concentration, $45 \mathrm{mg} / \mathrm{kg}$ body weight. Cur concentration, $2 \mathrm{mg} / \mathrm{kg}$ body weight. Ato concentration for the A-Lipo and AC-Lipo treatment groups, I.8 mg/kg body weight. Ato concentration for the T-AC-Lipo treatment group, $1.4 \mathrm{mg} / \mathrm{kg}$ body weight. (B) Illustration of E-selectin-targeting liposomes (T-AC-Lipo) simultaneously encapsulating Ato and Cur used to treat atherosclerotic ApoE knockout mice.

Abbreviations: Ato, atorvastatin calcium; A-Lipo, atorvastatin calcium-loaded liposome; AC-Lipo, atorvastatin calcium- and curcumin-loaded liposome; Cur, curcumin; MCP-I, monocyte chemotactic protein I; T-AC-Lipo, targeting-ligand-modified atorvastatin calcium- and curcumin-loaded liposome; WT, wild type.

\section{Discussion}

Statins, such as Ato, are first-line drugs for treatment of atherosclerosis; however, low bioavailability and adverse effects limit their widespread clinical application. To overcome these limitations, nanomedicine strategies have been developed to co-deliver drugs to achieve synergistic therapy and improve the bioavailability of hypolipidemic drugs through their solubilization effects. ${ }^{23-25} \mathrm{Cur}$ has been reported as beneficial in reduction of Ato-inducible toxicity ${ }^{10}$ and suppression of atherosclerosis, due to its antioxidative and anti-inflammatory activities.$^{51}$ Dysfunction of ECs induced by inflammatory factors and plasma lipids is closely associated with macrophage recruitment and lipid deposition in lesion sites, ${ }^{4}$ resulting in the occurrence and development of atherosclerosis. ${ }^{52}$ In the context of atherosclerosis, dysfunction of ECs is considered an initiating event. Suppression of adhesion molecules (eg, E-selectin and ICAM-1) in dysfunctional ECs could reduce adhesion and migration of monocytes into the intima, thereby blocking the formation of foam cells and the expression of inflammatory factors, such as IL-6 and MCP-1. Therefore, liposomes were prepared to co-deliver Ato and Cur to damaged ECs for synergistic anti-atherosclerosis therapy and reduction of Ato-induced cytotoxicity. Although the nanoscale particles, such as micelles, vesicles, and liposomes, were mainly captured by reticuloendothelial system, they could effectively accumulate at the plaques after injection via tail vein. ${ }^{47}$ The decoration with Esb peptide was able to endow liposome the inflamed ECs-targeted drug delivery.

Table 2 Plasma lipids, HMG-CoA reductase concentration, and weight of mice in each group after 30 days therapy with C-Lipo, A-Lipo, AC-Lipo, or T-AC-Lipo

\begin{tabular}{l|l|l|l|l|l}
\hline & TC $(\mathbf{m m o l} / \mathbf{L})$ & TG $(\mathbf{m m o l} / \mathbf{L})$ & LDL-C $(\mathbf{m m o l} / \mathbf{L})$ & HMG-CoA $(\mathbf{n g} / \mathbf{m L})$ & Weight $(\mathbf{g})$ \\
\hline WT & $5.27 \pm 0.84$ & $0.56 \pm 0.12$ & $0.47 \pm 0.26$ & $1.406 \pm 0.575$ & $23.4 \pm 1.09$ \\
Control & $21.26 \pm 1.91$ & $1.01 \pm 0.16$ & $7.20 \pm 0.69$ & $2.270 \pm 0.798$ & $28.0 \pm 0.84$ \\
C-Lipo & $17.15 \pm 0.36$ & $0.91 \pm 0.07$ & $5.67 \pm 0.70$ & $2.055 \pm 0.562$ & $27.5 \pm 0.98$ \\
A-Lipo & $12.63 \pm 1.41$ & $0.60 \pm 0.38$ & $4.34 \pm 0.88$ & $0.181 \pm 0.011$ & $25.9 \pm 1.23$ \\
AC-Lipo & $10.44 \pm 0.63$ & $0.53 \pm 0.11$ & $3.87 \pm 0.45$ & $0.171 \pm 0.026$ & $25.4 \pm 1.26$ \\
T-AC-Lipo & $8.09 \pm 0.79 *$ & $0.54 \pm 0.11$ & $2.68 \pm 0.39 *$ & $0.151 \pm 0.011$ & $26.0 \pm 1.82$ \\
\hline
\end{tabular}

Notes: HMG-CoA, HMG-CoA reductase. $* P<0.05$ vs control mouse group. WT, C57BL/6 mice. Control group, ApoE knockout mice.

Abbreviations: A-Lipo, atorvastatin calcium-loaded liposome; AC-Lipo, atorvastatin calcium- and curcumin-loaded liposome; C-Lipo, curcumin-loaded liposome; LDL-C, lowdensity lipoprotein cholesterol; T-AC-Lipo, targeting-ligand-modified atorvastatin calcium- and curcumin-loaded liposome; TC, total cholesterol; TG, triglyceride; WT, wild type. 
In addition, the $\mathrm{ApoE}^{-/-}$mouse model is extensively used for the evaluation of anti-atherosclerosis drugs, although its use is limited by the fact that its hyperlipidemic and atherosclerosis phenotypes are rescued by complementation with transplanted bone marrow from WT mice, in bone marrow transplantation studies. ${ }^{53}$

As expected, the synergistic anti-atherosclerosis effects of Ato and Cur were supported by our experimental data. Combined treatment with T-AC-Lipo reduced the percentage atherosclerotic area in the thoracic aorta in the control group, which is far superior than the results achieved with A-Lipo or C-Lipo treatment alone (Figure 6), consistent with the detected expression levels of inflammatory factors (Figure 8). Combination treatment with Ato and Cur also led to lower plasma levels of TC, TG, and LDL-C relative to those in animals treated with Ato or Cur alone (Table 2). Furthermore, the decoration of liposomes with targeting ligand resulted in superior anti-atherosclerosis effects and lower levels of inflammatory factors (E-selectin, ICAM-1, IL-6, and MCP-1) and plasma lipids (TC, TG, and LDL-C), strongly indicating that targeting ligand-modified liposomes could deliver substantially more Ato and Cur to ECs. For example, T-AC-Lipo treatment reduced the percentage atherosclerotic area to $17.14 \%$ from $39.69 \%$ of AC-Lipo treatment. These results are in agreement with previous reports that nanoparticles modified with antibody against E-selectin can significantly enhance internalization of drug into ECs. ${ }^{54,55}$ In addition, there was no significant difference between the A-Lipo and AC-Lipo treatment groups in the levels of E-selectin and ICAM-1 mRNA, which may be attributable to the lower cellular uptake efficiency of nontargeted treatment. In addition, Cur encapsulated in liposomes was previously reported to only induce anti-inflammatory effects at concentrations $>10 \mu \mathrm{g} / \mathrm{mL},{ }^{56}$ suggesting that the anti-inflammation and antioxidation effects of Cur are dose-dependent. Therefore, the liposomes modified with E-selectin-targeted ligand remarkably enhance the anti-atherosclerotic effects by increasing the intracellular concentration of Ato and Cur. Furthermore, T-AC-Lipo treatment increased cell viability to $70.12 \%$ from $55.72 \%$ in the A-Lipo treatment group, at an Ato concentration of $8 \mu \mathrm{g} / \mathrm{mL}$, which indicates that Cur delivered using targeting liposomes can effectively reduce Ato-induced cytotoxicity.

Although combination therapy with Ato and Cur achieved synergistic anti-atherosclerosis effects in the animal model, there is a considerable distance to bridge between these primary results and clinical application of this approach. Therefore, further studies, including investigation of blood circulation time and intracellular drug release, are required to optimize the therapeutic efficacy. The size and surface properties of the liposomes should be further optimized to prolong circulation time. ${ }^{57}$ Several approaches could be investigated to enhance the lysosomal escape ability of the liposomes. ${ }^{58,59}$ Targeting-ligand-modified liposomes could co-deliver Ato and Cur into dysfunctional ECs, resulting in enhanced anti-atherosclerosis effects via EC-specific drug delivery, combined with the synergistic activity of Ato and Cur. Hence, liposomes loaded with Ato and Cur could have similar clinical applications (eg, for lipid lowering and treatment of SAS) to those of Ato, but with increased therapeutic effects. ${ }^{11}$ To drive the Ato/Cur combination into the preclinical and clinical phases, we will conduct further experiments following the guidelines of the Food and Drug Administration, China.

\section{Conclusion}

Targeting liposomes to co-deliver Ato and Cur into dysfunctional ECs were generated. High cellular liposome uptake was achieved by Esb peptide-mediated endocytosis. Liposomes carrying both Ato and Cur could effectively inhibit the expression of adhesion (E-selectin, ICAM-1) and inflammatory (IL-6, MCP-1) molecules, and reduce plasma lipid levels, resulting in a significant decrease in atherosclerotic lesions. Co-delivery of Cur with the liposomes reduced the cytotoxicity induced by Ato. The results of both our in vitro and in vivo experiments demonstrate that targeted combination treatment with Ato and Cur produces far superior anti-atherosclerotic effects, relative to both nontargeted combination treatment and single-drug treatment (Ato or Cur alone), owing to the synergistic effects of the two therapeutic agents.

\section{Acknowledgments}

This work was supported by the National Key R\&D Program of China (2016YFE0117100), National Basic Research Program of China (2015CB755500), the National Natural Science Foundation of China (21875289, 51373203, U1501243, 31530023), Natural Science Foundation of the Guangdong Province (2014A030312018, 2015A030313283, 2016A030313554), the Guangdong Innovative and Entrepreneurial Research Team Program (2013S086), the GuangdongHongkong Joint innovation Project (2016A050503026), the Project on the Integration of Industry, Education and Research of Guangdong Province (2013B090500094), the Major Project on the Integration of Industry, Education and Research of Guangzhou City (201704030123), and the 
Fundamental Research Funds for the Central Universities (16lgjc59 and 20162900031650004).

\section{Disclosure}

The authors report no conflicts of interest in this work.

\section{References}

1. Libby P, Ridker PM, Maseri A. Inflammation and atherosclerosis. Circulation. 2002;105(9):1135-1143.

2. Linton MF, Fazio S. Macrophages, inflammation, and atherosclerosis. Int J Obes Relat Metab Disord. 2003;27 Suppl 3:S35-S40.

3. Lessner SM, Prado HL, Waller EK, Galis ZS. Atherosclerotic lesions grow through recruitment and proliferation of circulating monocytes in a murine model. Am J Pathol. 2002;160(6):2145-2155.

4. Manduteanu I, Simionescu M. Inflammation in atherosclerosis: a cause or a result of vascular disorders? J Cell Mol Med.2012;16(9):1978-1990.

5. Hansson GK. Inflammatory mechanisms in atherosclerosis. J Thromb Haemost. 2009;7(Suppl 1):328-331.

6. Wong WW-L, Dimitroulakos J, Minden MD, Penn LZ. HMG-CoA reductase inhibitors and the malignant cell: the statin family of drugs as triggers of tumor-specific apoptosis. Leukemia. 2002;16(4):508-519.

7. Taylor AJ, Kent SM, Flaherty PJ, Coyle LC, Markwood TT, Vernalis MN. ARBITER: Arterial biology for the investigation of the treatment effects of reducing cholesterol: a randomized trial comparing the effects of atorvastatin and pravastatin on carotid intima medial thickness. Circulation. 2002;106(16):2055-2060.

8. Aydin U, Ugurlucan M, Gungor F, et al. Effects of atorvastatin on vascular intimal hyperplasia: an experimental rodent model. Angiology. 2009;60(3):370-377.

9. Thompson PD, Panza G, Zaleski A, Taylor B. Statin-associated side effects. J Am Coll Cardiol. 2016;67(20):2395-2410.

10. Elshama SS, El-Kenawy AE-M, Osman H-EH. Curcumin improves atorvastatin-induced myotoxicity in rats: histopathological and biochemical evidence. Int J Immunopathol Pharmacol. 2016;29(4):742-752.

11. Sahebkar A, Saboni N, Pirro M, Banach M. Curcumin: an effective adjunct in patients with statin-associated muscle symptoms? J Cachexia Sarcopenia Muscle. 2017;8(1):19-24.

12. Banach M, Patti AM, Giglio RV, et al. The role of nutraceuticals in statin intolerant patients. J Am Coll Cardiol. 2018;72(1):96-118.

13. Michalska-Kasiczak M, Sahebkar A, Mikhailidis DP, et al. Analysis of vitamin D levels in patients with and without statin-associated myalgia a systematic review and meta-analysis of 7 studies with 2420 patients. Int J Cardiol. 2015;178:111-116.

14. Anand P, Kunnumakkara AB, Newman RA, Aggarwal BB. Bioavailability of curcumin: problems and promises. Mol Pharm. 2007;4(6): 807-818.

15. Aggarwal BB, Harikumar KB. Potential therapeutic effects of curcumin, the anti-inflammatory agent, against neurodegenerative, cardiovascular, pulmonary, metabolic, autoimmune and neoplastic diseases. Int $J$ Biochem Cell Biol. 2009;41(1):40-59.

16. Aggarwal BB, Kumar A, Bharti AC. Anticancer potential of curcumin: preclinical and clinical studies. Anticancer Res. 2003;23(1A):363-398.

17. Simental-Mendía LE, Pirro M, Gotto AM, et al. Lipid-modifying activity of curcuminoids: a systematic review and meta-analysis of randomized controlled trials. Crit Rev Food Sci Nutr. 2017;40:1-10.

18. Ganjali S, Blesso CN, Banach M, Pirro M, Majeed M, Sahebkar A. Effects of curcumin on HDL functionality. Pharmacol Res. 2017;119: 208-218.

19. Olszanecki R, Jawień J, Gajda M, et al. Effect of curcumin on atherosclerosis in apoE/LDLR-double knockout mice. J Physiol Pharmacol. 2005;56(4):627-635.

20. Sahebkar A, Kotani K, Serban C, et al. Statin therapy reduces plasma endothelin-1 concentrations: a meta-analysis of 15 randomized controlled trials. Atherosclerosis. 2015;241(2):433-442.
21. Serban C, Sahebkar A, Ursoniu S, Mikhailidis DP, Rizzo M. A systematic review and meta-analysis of the effect of statins on plasma asymmetric dimethylarginine concentrations. Sci Rep. 2015;241:199.

22. Khadka P, Ro J, Kim H, et al. Pharmaceutical particle technologies: an approach to improve drug solubility, dissolution and bioavailability. Asian J Pharm. 2014;9(6):304-316.

23. Kalashnikova I, Mazar J, Neal CJ, et al. Nanoparticle delivery of curcumin induces cellular hypoxia and ROS-mediated apoptosis via modulation of Bcl-2/Bax in human neuroblastoma. Nanoscale. 2017;9(29): 10375-10387.

24. Xu P, Yu H, Zhang Z, et al. Hydrogen-bonded and reduction-responsive micelles loading atorvastatin for therapy of breast cancer metastasis. Biomaterials. 2014;35(26):7574-7587.

25. Alupei MC, Licarete E, Patras L, Banciu M. Liposomal simvastatin inhibits tumor growth via targeting tumor-associated macrophagesmediated oxidative stress. Cancer Lett. 2015;356(2 Pt B):946-952.

26. Malam Y, Loizidou M, Seifalian AM. Liposomes and nanoparticles: nanosized vehicles for drug delivery in cancer. Trends Pharmacol Sci. 2009; 30(11):592-599.

27. Yin $\mathrm{T}$, Wang $\mathrm{P}, \mathrm{Li} \mathrm{J}$, et al. Tumor-penetrating codelivery of siRNA and paclitaxel with ultrasound-responsive nanobubbles hetero-assembled from polymeric micelles and liposomes. Biomaterials. 2014;35(22):5932-5943.

28. He C, Tang Z, Tian H, Chen X. Co-delivery of chemotherapeutics and proteins for synergistic therapy. Adv Drug Deliv Rev. 2016;98:64-76.

29. Takara K, Hatakeyama H, Kibria G, Ohga N, Hida K, Harashima H. Size-controlled, dual-ligand modified liposomes that target the tumor vasculature show promise for use in drug-resistant cancer therapy. J Control Release. 2012;162(1):225-232.

30. Liu J, Jiang X, Ashley C, Brinker CJ. Electrostatically mediated liposome fusion and lipid exchange with a nanoparticle-supported bilayer for control of surface charge, drug containment, and delivery. $J \mathrm{Am}$ Chem Soc. 2009;131(22):7567-7569.

31. Sapra P, Tyagi P, Allen T. Ligand-targeted liposomes for cancer treatment. Curr Drug Delivery. 2005;2(4):369-381.

32. Stefanick JF, Ashley JD, Kiziltepe T, Bilgicer B. A systematic analysis of peptide linker length and liposomal polyethylene glycol coating on cellular uptake of peptide-targeted liposomes. ACS Nano. 2013;7(4):2935-2947.

33. Yang Y, Yang Y, Xie X, et al. Dual-modified liposomes with a twophoton-sensitive cell penetrating peptide and NGR ligand for siRNA targeting delivery. Biomaterials. 2015;48:84-96.

34. Chen C, Duan Z, Yuan Y, et al. Peptide-22 and cyclic RGD functionalized liposomes for glioma targeting drug delivery overcoming BBB and BBTB. ACS Appl Mater Interfaces. 2017;9(7):5864-5873.

35. Everts M, Schraa AJ, de Leij L, Meijer DKF, Molema G. Vascular endothelium in inflamed tissue as a target for site selective delivery of drugs. In: Molema G, Meijer DKF, editors. Drug Targeting: OrganSpecific Strategies. Weinheim: Wiley-VCH; 2001;12:171-197.

36. Leus NG, Talman EG, Ramana P, et al. Effective siRNA delivery to inflamed primary vascular endothelial cells by anti-E-selectin and anti-VCAM-1 PEGylated SAINT-based lipoplexes. Int J Pharm. 2014; 459(1-2):40-50.

37. Morane LH, Ly T, Nicolas R. Proinflammatory signature of the dysfunctional endothelium in pulmonary hypertension. Role of the macrophage migration inhibitory factor/CD74 complex. Am J Resp Crit Care. 2015;192(8):983-997.

38. Jubeli E, Moine L, Vergnaud-Gauduchon J, Barratt G. E-selectin as a target for drug delivery and molecular imaging. J Control Release. 2012;158(2):194-206.

39. Bhaskar V, Law DA, Ibsen E, et al. E-selectin up-regulation allows for targeted drug delivery in prostate cancer. Cancer Res. 2003;63(19):6387-6394.

40. Ghalandarlaki N, Alizadeh AM, Ashkani-Esfahani S. Nanotechnologyapplied curcumin for different diseases therapy. Biomed Res Int. 2014; 3:394264.

41. Kumar N, Chaurasia S, Patel RR, Kumar V, Mishra B. Development and optimization of atorvastatin calcium loaded oral biodegradable polymeric nanoparticles using central composite design. Adv Sci Lett. 2014; 20(5):984-993. 
42. Gu L, Okada Y, Clinton SK, et al. Absence of monocyte chemoattractant protein-1 reduces atherosclerosis in low density lipoprotein receptordeficient mice. Mol Cell. 1998;2(2):275-281.

43. Michelsen KS, Wong MH, Shah PK, et al. Lack of Toll-like receptor 4 or myeloid differentiation factor 88 reduces atherosclerosis and alters plaque phenotype in mice deficient in apolipoprotein E. Proc Natl Acad Sci U S A. 2004;101(29):10679-10684.

44. Mann AP, Tanaka T, Somasunderam A, Liu X, Gorenstein DG, Ferrari M. Site-specific drug delivery: E-selectin-targeted porous silicon particle for nanoparticle delivery to the bone marrow. Adv Mater. 2011; 23(36):H284.

45. Deguchi JO, Aikawa M, Tung $\mathrm{CH}$, et al. Inflammation in atherosclerosis: visualizing matrix metalloproteinase action in macrophages in vivo. Circulation. 2006;114(1):55-62.

46. Li C, Zhang WJ, Frei B. Quercetin inhibits LPS-induced adhesion molecule expression and oxidant production in human aortic endothelial cells by p38-mediated Nrf2 activation and antioxidant enzyme induction. Redox Biol. 2016;9:104-113.

47. Wu T, Chen X, Wang Y, et al. Aortic plaque-targeted andrographolide delivery with oxidation-sensitive micelle effectively treats atherosclerosis via simultaneous ROS capture and anti-inflammation. NanomedNanotechnol. 2018;14(7):2215-2226.

48. Paoletti R, Gotto AM Jr, Hajjar DP. Inflammation in atherosclerosis and implications for therapy. Circulation. 2004;109(23 Suppl 1): III-20-III-26.

49. Viedt C, Vogel J, Athanasiou T, et al. Monocyte chemoattractant protein-1 induces proliferation and interleukin- 6 production in human smooth muscle cells by differential activation of nuclear factor-kappaB and activator protein-1. Arterioscler Thromb Vasc Biol. 2002;22(6): 914-920.
50. Hasan ST, Zingg J-M, Kwan P, Noble T, Smith D, Meydani M. Curcumin modulation of high fat diet-induced atherosclerosis and steatohepatosis in LDL receptor deficient mice. Atherosclerosis. 2014;232(1): 40-51.

51. Vallianou NG, Evangelopoulos A, Schizas N, Kazazis C. Potential anticancer properties and mechanisms of action of curcumin. Anticancer Res. 2015;35(2):645.

52. Hansson GK. Immune and inflammatory mechanisms in the development of atherosclerosis. Br Heart J. 1993;69(1 Suppl):S38-S41.

53. Emini Veseli B, Perrotta P, de Meyer GRA, et al. Animal models of atherosclerosis. Eur J Pharmacol. 2017;816:3-13.

54. Gholizadeh S, Visweswaran G, Storm G, Hennink WE, Kamps J, Kok RJ. E-selectin targeted immunoliposomes for rapamycin delivery to activated endothelial cells. Int J Pharmaceut. 2017;54(2):759-770.

55. Ma S, Tian XY, Zhang Y, et al. E-selectin-targeting delivery of microRNAs by microparticles ameliorates endothelial inflammation and atherosclerosis. Sci Rep. 2016;6(1):22910.

56. Basnet P, Hussain H, Tho I, Skalko-Basnet N. Liposomal delivery system enhances anti-inflammatory properties of curcumin. J Pharm Sci. 2012;101(2):598-609.

57. Moghimi SM, Szebeni J. Stealth liposomes and long circulating nanoparticles: critical issues in pharmacokinetics, opsonization and protein-binding properties. Prog Lipid Res. 2003;42(6):463-478.

58. Li Y, Cheng Q, Jiang Q, et al. Enhanced endosomal/lysosomal escape by distearoyl phosphoethanolamine-polycarboxybetaine lipid for systemic delivery of siRNA. J Control Release. 2014;176(1):104-114.

59. Li Y, Liu R, Shi Y, Zhang Z, Zhang X, Ruiyuan L. Zwitterionic poly(carboxybetaine)-based cationic liposomes for effective delivery of small interfering RNA therapeutics without accelerated blood clearance phenomenon. Theranostics. 2015;5(6):583-596. 


\section{Supplementary material}

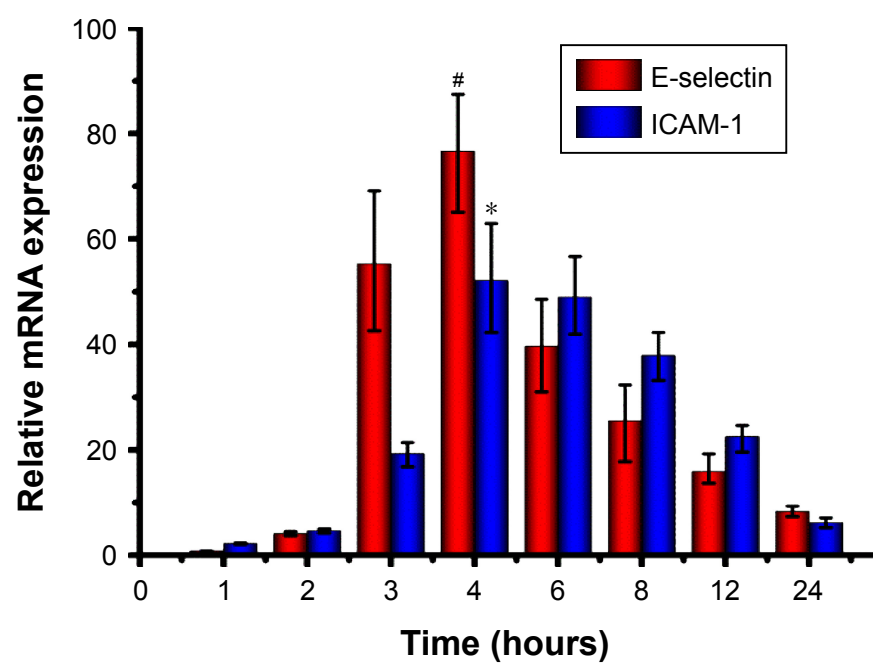

Figure SI Relative gene expression levels of E-selectin and ICAM-I in human aortic endothelial cells stimulated with I $\mu \mathrm{g} / \mathrm{mL}$ lipopolysaccharide for different periods of time (mean $\pm \mathrm{SD} ; \mathrm{n}=3 ; * P<0.05$ and $\# \mathbb{P}<0.05$ compared with that of 2 hours).

\section{Publish your work in this journal}

The International Journal of Nanomedicine is an international, peerreviewed journal focusing on the application of nanotechnology in diagnostics, therapeutics, and drug delivery systems throughou the biomedical field. This journal is indexed on PubMed Central, MedLine, CAS, SciSearch $®$, Current Contents $\AA /$ Clinical Medicine,
Journal Citation Reports/Science Edition, EMBase, Scopus and the Elsevier Bibliographic databases. The manuscript management system is completely online and includes a very quick and fair peer-review system, which is all easy to use. Visit http://www.dovepress.com/ testimonials.php to read real quotes from published authors. 